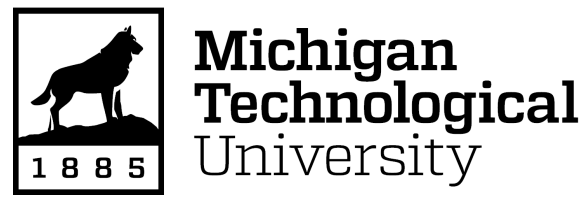

Michigan Technological University Digital Commons @ Michigan Tech

Dissertations, Master's Theses and Master's Reports

2021

\title{
THE INFLUENCE OF DECREASED AMBIENT LIGHTING ON REACTIVE BALANCE MECHANISMS IN YOUNG ADULTS
}

Jessica Pitts

Michigan Technological University, jpitts@mtu.edu

Copyright 2021 Jessica Pitts

Recommended Citation

Pitts, Jessica, "THE INFLUENCE OF DECREASED AMBIENT LIGHTING ON REACTIVE BALANCE MECHANISMS IN YOUNG ADULTS", Open Access Master's Thesis, Michigan Technological University, 2021.

https://doi.org/10.37099/mtu.dc.etdr/1249

Follow this and additional works at: https://digitalcommons.mtu.edu/etdr 


\title{
THE INFLUENCE OF DECREASED AMBIENT LIGHTING ON REACTIVE BALANCE MECHANISMS IN YOUNG ADULTS
}

\author{
By \\ Jessica L. Pitts

\begin{abstract}
A THESIS
Submitted in partial fulfillment of the requirements for the degree of MASTER OF SCIENCE

In Kinesiology
\end{abstract}

MICHIGAN TECHNOLOGICAL UNIVERSITY

2021

(C) 2021 Jessica L. Pitts 
This thesis has been approved in partial fulfillment of the requirements for the Degree of MASTER OF SCIENCE in Kinesiology.

Department of Kinesiology and Integrative Physiology

Thesis Advisor: $\quad$ Dr. Carolyn Duncan

Committee Member:

Dr. Kevin Trewartha

Committee Member:

Dr. Shane Mueller

Committee Member:

Dr. Tanvi Bhatt

Department Chair:

Dr. Megan Frost 


\section{Table of Contents}

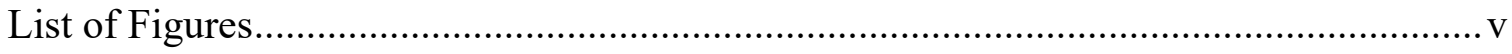

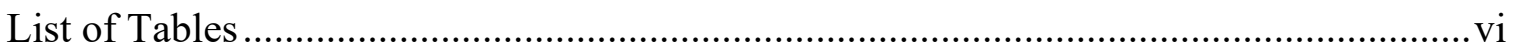

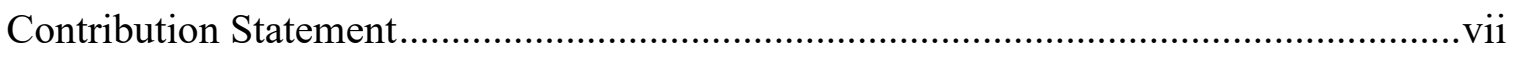

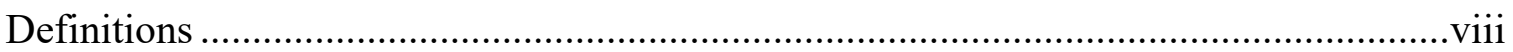

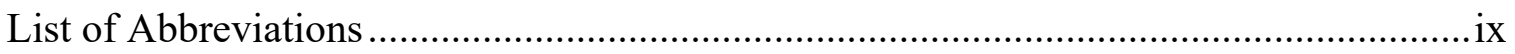

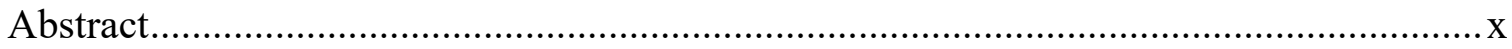

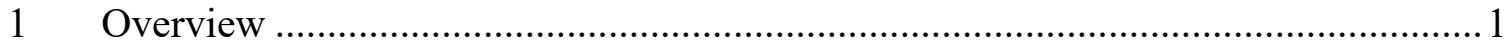

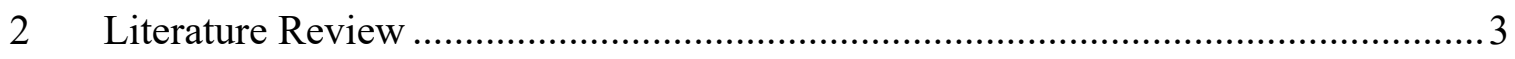

2.1 General Models of Balance Control............................................................. 3

2.2 Sensorimotor Control of Balance......................................................... 4

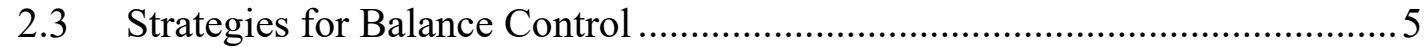

2.3.1 Fixed-Support Strategies .......................................................... 7

2.3.2 Change-in-Support Strategies ......................................................... 8

2.4 Factors Affecting Postural Control and Fall Risk ..................................... 9

2.4.1 Investigating Fall Risk through Balance Recovery ............................ 10

2.5 Visual Contributions to Balance Control .................................................... 12

2.5.1 Vision and Anticipatory Balance ................................................. 12

2.5.2 Vision and Gait ............................................................................... 14

2.5.3 Vision and Reactive Balance ...................................................... 14

2.6 Environmental Lighting and Balance Control .......................................... 17

2.7 Remaining Questions from the Literature.............................................. 19

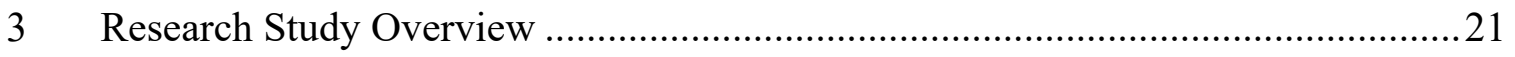

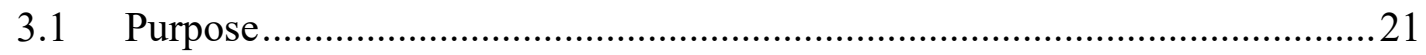

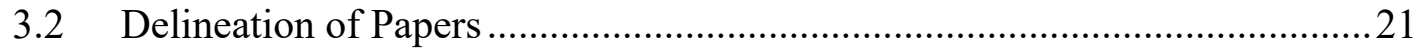

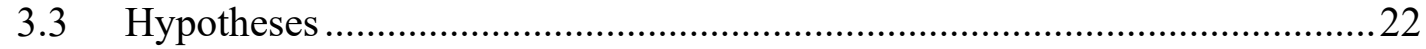

4 Paper 1 - The effect of decreased ambient lighting on reactive stepping kinematics and center of mass control following forward losses of balance ...................................24

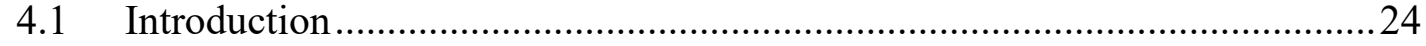

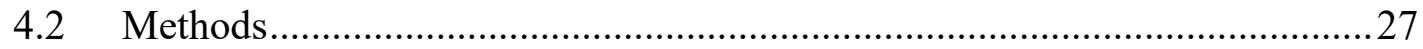

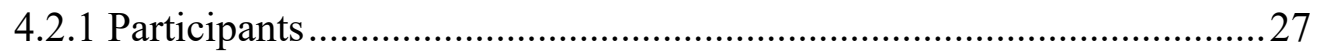

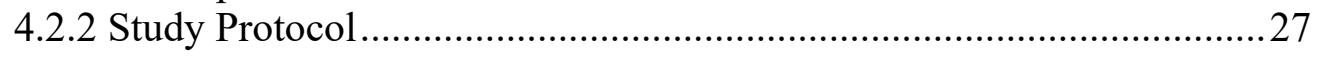

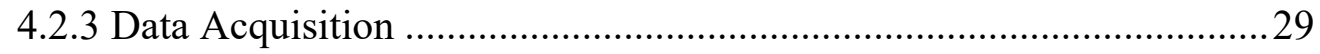

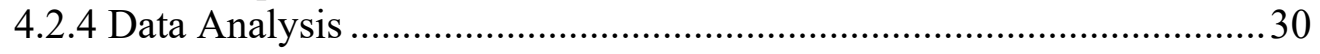

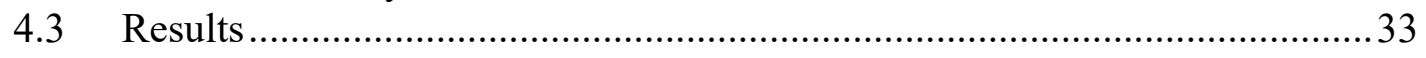




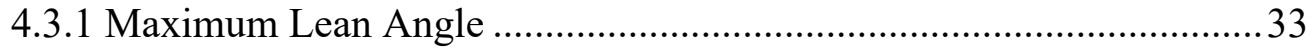

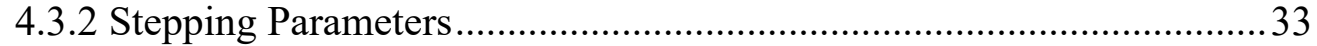

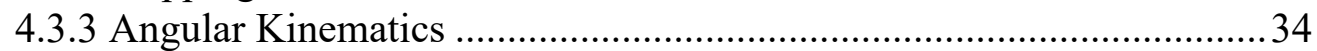

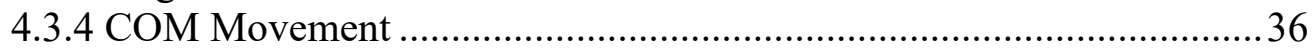

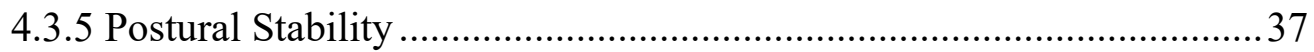

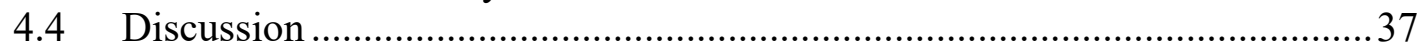

5 Paper 2 - The effect of decreased ambient lighting on neuromuscular responses while anticipating and responding to forward losses of balance ........................................4

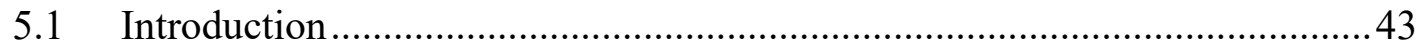

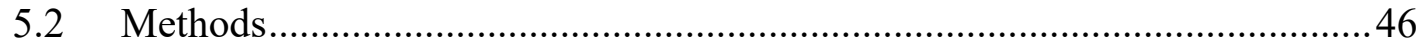

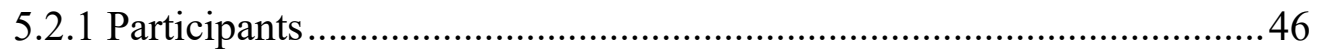

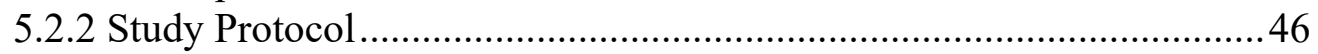

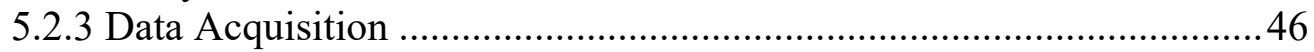

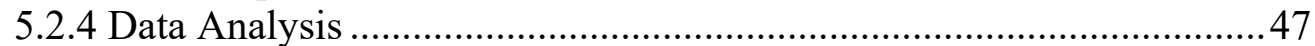

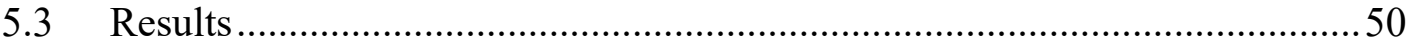

5.3.1 Muscle Onset Latencies .................................................................50

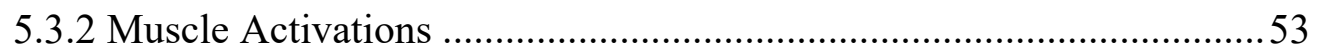

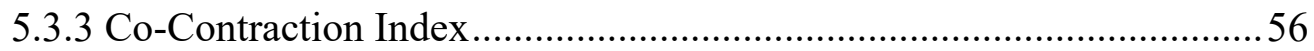

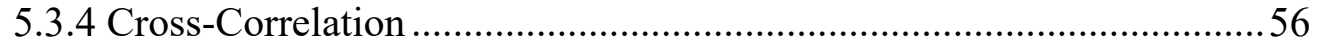

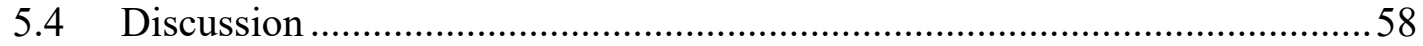

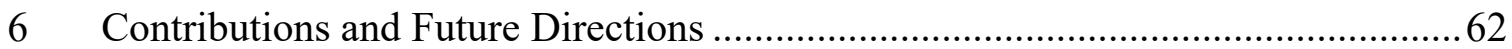

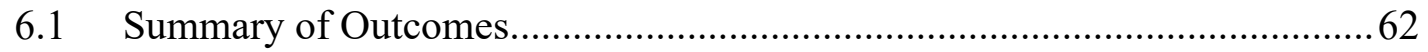

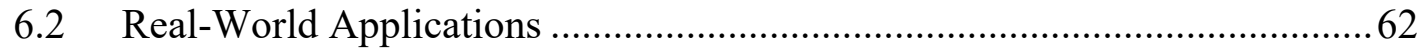

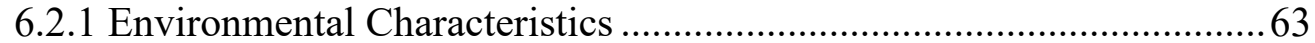

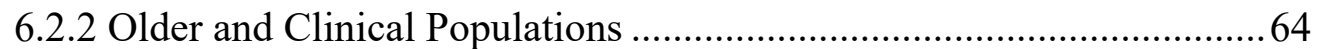

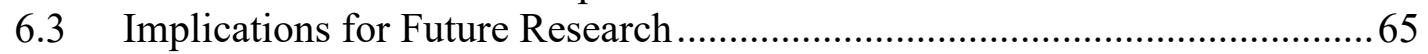

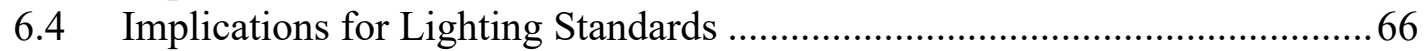

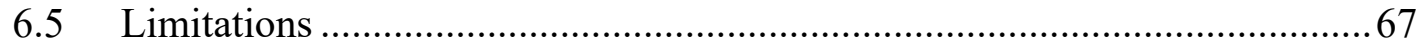

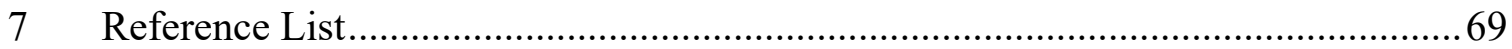

A Participant Eligibility Checklist .......................................................................

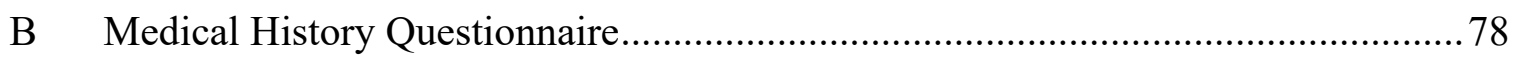

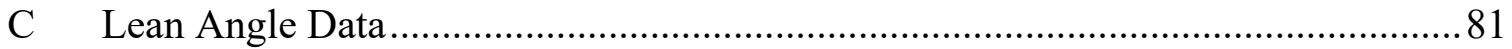

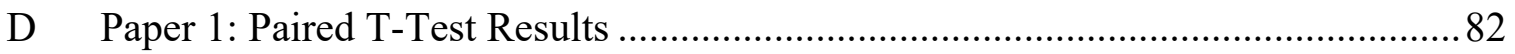

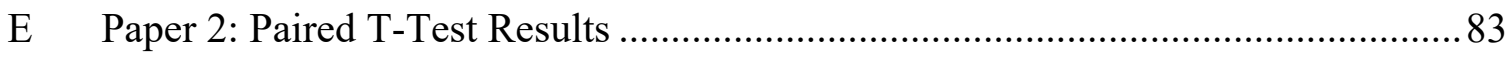




\section{List of Figures}

Figure 2.1 Balance recovery using fixed-support and change-in-support strategies.... 8

Figure 2.2 Factors contributing to postural control and fall risk

Figure 2.3 Individual, environmental, and task factors that influence balance recovery ability

Figure 4.1 (A) Lean-and-Release Fall Arrest System with coordinate system 28

Figure 4.2 Step length (A), height (B), width (C), velocity (D), time (E), and onset time (F) in different lighting conditions 34

Figure 4.3 Peak +/- angular velocity at the ankle, knee, hip, and trunk 35

Figure 4.4 (A) Peak COM displacement, velocity, and acceleration 36

Figure 4.5 $\mathrm{X}_{\mathrm{COM} / \mathrm{BOS}}$ values in lighting conditions presented as a percentage of step length in A-P direction and step width in M-L direction

Figure 5.1 (A) Muscle onset latencies for tibialis anterior (TA), medial gastrocnemius $(\mathrm{MG})$, rectus femoris (RF), and biceps femoris (BF)

Figure 5.2 Mean Activation of the tibialis anterior (TA), medial gastrocnemius (MG), rectus femoris $(\mathrm{RF})$, and biceps femoris $(\mathrm{BF})$

Figure 5.3 Maximum activation of the tibialis anterior (TA), medial gastrocnemius $(\mathrm{MG})$, rectus femoris $(\mathrm{RF})$, and biceps femoris $(\mathrm{BF})$

Figure 5.4 Co-Contraction Index (CCI) in the shank segment (TA-MG), thigh segment (RF-BF), anterior muscles (TA-RF), and posterior muscles (MG-BF)..........56

Figure 5.5 Variability in zero-lag cross-correlation coefficients between shank (TA-MG), thigh (RF-BF), anterior (TA-RF), and posterior (MG-BF) muscles

Figure 6.1 Individual, environmental, and task factors that influence balance recovery ability 


\section{List of Tables}

Table 3.1 Breakdown of Thesis Papers ...................................................................23

Table 6.1 Proposed addition of dim lighting to environmental balance recovery factors

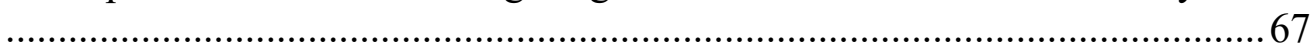




\section{Contribution Statement}

This report was collaboratively developed by myself and my advisor, Dr. Carolyn Duncan. I conducted a review of the existing literature, collected data for the reported study, and prepared this report. Dr. Duncan and I worked together to edit and improve the presentation of this report. We plan to submit the two papers presented in this thesis as journal articles in the near future. Co-authors Dr. Alicia DenHerder, Dr. Vicki Komisar, and Dr. Alison Schinkel-Ivy assisted in the editing of chapters 4 and 5. 


\section{Definitions}

Balance. Balance refers to a state in which the body is positioned so that the center of mass $(\mathrm{COM})$ is within the areas of the body in contact with the ground (base of support) [1].

Balance Recovery. Balance recovery, also known as reactive balance, is an action of the body that aims to restore upright stance following a perturbation by re-stabilizing the COM.

Base of Support (BOS). The body's base of support refers to the areas of the body, generally the feet, which are in contact with the ground. The arms can also be included in the base of support when applying forces to external surfaces or handrails.

Center of Mass (COM). Center of mass is a point representing the average location and total mass of the entire body, derived from the location and mass of each individual body segment.

Middle-Aged Adults. Middle-aged adults are between the ages of 40 and 64 years old.

Older Adults. Older adults are 65 years old and above.

Perturbation. A perturbation is an internally or externally generated force on the body that threatens balance by destabilizing the COM.

Postural Control. Postural control is the process of controlling the COM over the BOS.

Young Adults. Young adults are between the ages of 18-39 years old.

viii 


\section{List of Abbreviations}

A-P: Anterior-Posterior

BF: Biceps Femoris

BOS: Base of Support

CNS: Central Nervous System

COM: Center of Mass

MG: Medial Gastrocnemius

M-L: Medial-Lateral

RF: Rectus Femoris

TA: Tibialis Anterior 


\section{Abstract}

Introduction: Balance recovery/reactive balance prevents falls by restabilizing the center of mass (COM) when instability occurs. Dim lighting is a risk factor for falling, although limited research has examined how lighting conditions affect the ability to recover from losses of balance. The purpose of this thesis was to examine the effect of lighting conditions on reactive balance mechanisms. Methods: 20 young adults (23.3(4.4) y) completed forward lean-and-release perturbations in two lighting conditions: 1) Light: 800 Lux; 2) Dark: 0 Lux. Optical motion capture and surface electromyography were used to quantify stepping/angular kinematics, COM control, and the timing, activation, and coordination of lower-limb neuromuscular responses. Differences between lighting conditions were analyzed with paired sample t-tests (alpha=0.05). Results: In darkness, individuals showed modified stepping (increased step length, knee flexion/extension velocity, and hip flexion velocity $(\mathrm{p}<0.05)$ ), decreased COM control (increased trunk extension velocity and decreased postural stability $(\mathrm{p}<0.05))$, and changes to neuromuscular responses (increased tibialis anterior activation and thigh coordination $(\mathrm{p}<0.05))$. Individuals showed no differences in response timing or recovery ability. Discussion: Balance recovery in dark environments can result in modified stepping responses and decreased COM control, which could prevent successful balance recovery in real-world environments and populations. Evidence-based lighting standards could minimize fall risk in built environments and public spaces. 


\section{Overview}

Adults reported an estimated 80 million falls in the United States in 2016, resulting in 3.2 million injuries, and making falls the leading cause of unintentional injuries [2]. Fall-related injuries are most commonly fractures and abrasions, but can also include lacerations, sprains, and internal injuries which result in substantial costs of treatment, hospitalizations, operative procedures, and fatalities [3]. Additionally, the percentage of fall-related injuries, hospital admissions, and deaths in the elderly have been increasing yearly since 2010 [4]. Balance research is critical to understand how the body reacts to instability, identify risk factors and environmental concerns that increase fall incidence, and ideally improve balance in individuals who are at a high risk for falls and associated injuries.

The human body is prone to falls due to its inherent instability [5]. Most of the body's weight is carried high off the ground towards the head, which must be stabilized over a narrow base of ground contact while maneuvering an unpredictable environment [5]. Postural control involves maintaining this body weight in a stable position (postural stability) based on the awareness of body position in space (postural orientation) [6]. Maintaining stability requires a combination of anticipatory feedback, in order to predict instances of instability, and reactive feedback, to respond to instability when it occurs [7]. Gravity, losses of balance, and other perturbing forces constantly challenge postural control by threatening to move the body weight away from a stable position. In order to prevent falls, the postural control system must be able to anticipate these instances of instability, rapidly detect postural deviations, and respond with corrective motor responses before body weight has moved outside of a recoverable range. Perturbing 
forces can be generated internally (e.g., limb movement and other changes in body position) or externally (e.g., a push on the body) and vary in timing, magnitude, and direction, making instances of instability temporally and characteristically unpredictable. When preemptive or reactive control is inhibited by a lack of time or insufficient motor response, falls can occur with detrimental results.

Dim lighting is a risk factor for falling [8] and is commonly experienced in daily life (e.g., dark bedroom after turning off lights, dimly lit movie theater, parking lot at night). Dark environments decrease the availability of visual information, which can negatively affect postural control and the ability to recover from perturbations $[9,10]$. Many falls are reported in areas of dim lighting [11, 12], however limited research has examined how decreased lighting affects balance control mechanisms, particularly how the body recovers from losses of balance. This thesis examined the effects of decreased ambient lighting on balance recovery mechanisms when responding to large perturbations. It is crucial to examine how dark environments affect the ability to prevent falls in order to understand why falls occur in dark environments and how they could be reduced. 


\section{Literature Review}

The purpose of this thesis was to explore how lighting conditions affect balance recovery ability and mechanisms. This literature review will summarize the basic principles of balance control (Section 2.1: General Models of Balance Control, 2.2: Sensorimotor Control of Balance, 2.3: Strategies for Balance Control) and analyze how different factors can affect the effectiveness of balance recovery (2.4: Factors affecting Balance Recovery and Fall Risk, 2.5: Aging and Balance Control). I then summarize what is known about balance recovery in conditions where vision is removed (2.6 Visual Contributions to Balance Control) and how these findings could apply to balance recovery in dark environments (2.7 Environmental Lighting and Balance Control, 2.8 Remaining Questions from the Literature).

\subsection{General Models of Balance Control}

The body is naturally unstable due to carrying two-thirds of body weight in the top third of body height and requires a constant control system to maintain stability [5]. Postural control is responsible for controlling body orientation (the position of body segments relative to each other and the environment) [13] and stability (the location of the center of mass (COM) relative to the base of support (BOS)) [6]. The COM is a single point that represents the weighted location and mass of all body segments [5] and must be controlled relative to the BOS (the areas of the body in contact with the ground) to maintain stability. The position of the COM is believed to be the key variable controlled during postural control [14], however ideal body orientation and COM position change relative to the required task [13]. While standing, the COM must be controlled relative to 
the limits of stability defined by the BOS to maintain an upright stance, based on sensory information regarding body position and postural deviations.

\subsection{Sensorimotor Control of Balance}

Dark environments could affect the ability to control the COM and maintain upright stance by altering sensory information. In order to maintain balance, the central nervous system (CNS) must be aware of how the body is positioned in space and determine when body position is altered due to instability. The postural control system integrates and organizes sensory information from the visual, vestibular, and somatosensory systems to track body position and sense linear and rotational movement [5]. These senses are responsible for perceiving self-motion and detecting deviations from upright stance [15]. When postural destabilization begins, the vestibular system signals changes in head and eye position relative to gravity, the visual system provides a reference to the external world, and the somatosensory system senses joint and muscle movement $[6,16]$. These feedback inputs allow the CNS to plan and implement an appropriate corrective muscular response based on the perturbation characteristics [16].

Sensory inputs from all three systems are not guaranteed to be available or accurate. For example, visual information is not as available in darkness, and somatosensory information is not as accurate when standing on an uneven or soft surface. Likewise, individuals may have physical or neurological impairment (e.g., vestibular disorders, blindness, peripheral neuropathy, etc.) that limit or affect the accuracy of sensory inputs. In these instances, the postural control system must adapt to consistently maintain stability [17]. Many researchers agree that humans rely on an optimal 
combination of sensory inputs from these three systems in different scenarios [18]. The most accurate and readily available senses contribute the most to sensorimotor integration and the senses which are missing information or are less accurate, contribute less [19].

The process wherein the CNS 'weights' the input from each sensory system is referred to as sensory weighting, or if the CNS is re-assigning weights, sensory reweighting [20]. Sensory reweighting allows individuals to maintain balance in a variety of situations by modifying the contribution of different senses for postural control. For example, individuals with vestibular disorders can rely on visual and somatosensory information to maintain postural equilibrium because their vestibular information is unreliable [21]. Peterka (2002) found healthy adults to rely $70 \%$ on somatosensory information, $20 \%$ on vestibular information, and $10 \%$ on visual information during standing balance with no challenges to sensory inputs [17]. The weighted contribution of each sensory system may depend on the processing speed of the system. Somatosensory processing (e.g., detecting a change in support surface contact) occurs much faster than visual and vestibular processing. Muscle response latencies to somatosensory indicators of postural instability are $80-100 \mathrm{~ms}$, but $200 \mathrm{~ms}$ when responding to visual indicators [22]. Rapid muscular responses to instability are key when preventing a fall, therefore the initial response following instability may rely mainly on somatosensory information. However, visual information may contribute more to balance later in the response [6].

\subsection{Strategies for Balance Control}

Balance control can be divided into two categories based on the temporal relationship between the perturbation and the motor response: 1) Anticipatory Balance 
and 2) Reactive Balance. Anticipatory balance involves maintaining stability in anticipation of an external perturbation by making small, feedforward adjustments in the body to keep the COM within the limits of stability preceding any instability [5]. My thesis was primarily focused on reactive balance, therefore anticipatory balance will not be discussed further. For more information on anticipatory balance control please refer to reviews by Winter (1995) [5] and Massion (1992) [23].

The purpose of this thesis was to examine if dark environments affect the ability to recover from losses of balance or the mechanics involved during reactive balance. Reactive balance (or balance recovery) involves regaining stability in response to a destabilizing perturbation. While anticipatory balance is important in maintaining posture during daily instabilities (e.g., gait, sit-to-stand, or climbing stairs), reactive balance is necessary to prevent falls when individuals encounter unpredictable perturbations that cannot be anticipated, such as a push, trip, or slip. When a perturbation forces the COM outside the limits of stability, reactive balance is responsible for producing a rapid motor response which returns the COM to a stable position in order to prevent a fall [24]. The $\mathrm{COM}$ can be re-stabilized by expanding the BOS to accommodate a larger range of stability for the COM or producing joint torques which move the COM closer to the existing BOS [25]. These strategies can utilize both the arms (e.g., rapid arm movement to counteract COM movement, grabbing a hand railing) and the trunk and legs (e.g., contracting leg and hip musculature, stepping away from the existing BOS). The motor responses of reactive balance can be classified as ankle, hip, step, and reach-to-grasp strategies. 
Reactive movements are fundamentally different from volitional movements in their timing. Following a perturbation, the planning and execution of postural muscle activation must occur very rapidly to regain control of the COM before a fall occurs [24]. Reactive movements typically occur twice as fast as the most rapid volitional movements ( $\sim 100 \mathrm{~ms}$ reduction in activation latency), even given the complexity of the response [26]. This muscular response must account for perturbation characteristics (e.g., speed, direction) and environmental layout (e.g., handrail location, barriers to stepping) without any pre-planning when the perturbation is unexpected [6]. These motor responses can be categorized into two main types: 1) Fixed-Support Strategies (ankle, hip) and 2) Changein-Support Strategies (step, reach-to-grasp) (Figure 2.1) [24, 27].

\subsubsection{Fixed-Support Strategies: Fixed-support strategies stabilize the COM} by producing muscle torques at the ankle (ankle strategy) or hip (hip strategy) to move the COM closer to the upright position while keeping the BOS unchanged [24]. Using the ankle strategy, an individual produces muscle activity that moves the body primarily at the ankle joint, either plantarflexing to move the COM posteriorly or dorsiflexing to move the COM anteriorly, depending on the direction of the perturbation. For example, following a perturbation that causes the COM to move anteriorly, an individual would plantarflex to counteract this momentum and move the COM in the opposite direction.

Similarly, an individual can use the hip strategy to either flex or extend the hip to move the COM anteriorly or posteriorly. The hip strategy is capable of producing larger stabilizing torques than the ankle strategy due to larger cross-sectional areas in the hip musculature and longer moment arms than the ankle. Therefore, the hip strategy can 
correct for stronger perturbations than the ankle strategy [28]. However, it is common for individuals to employ strategies that lie on a continuum between ankle and hip strategies, rather than distinctly utilizing one or the other [6]. The arms can also play a role in COM stabilization, even without contacting an external surface. Following a perturbation, muscles in the arms activate after a very short onset latency [29], producing rapid counteractive movements that can decrease the forward momentum of the body $[30,31]$ and reduce recovery time [32].

Fixed-Support Strategies

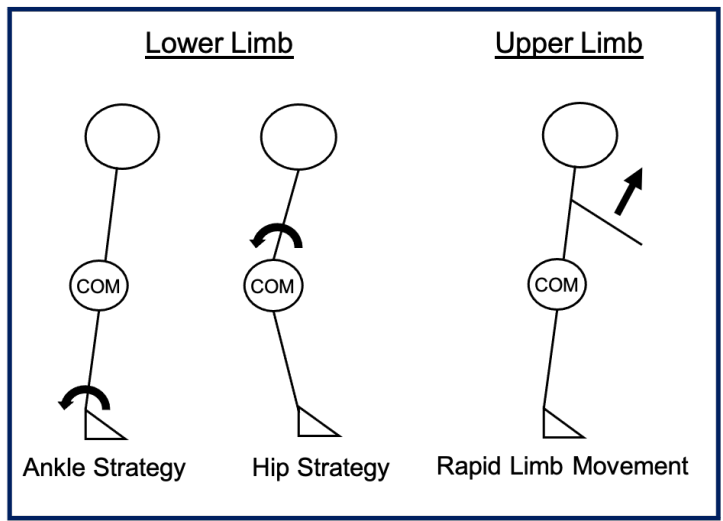

Change-in-Support Strategies

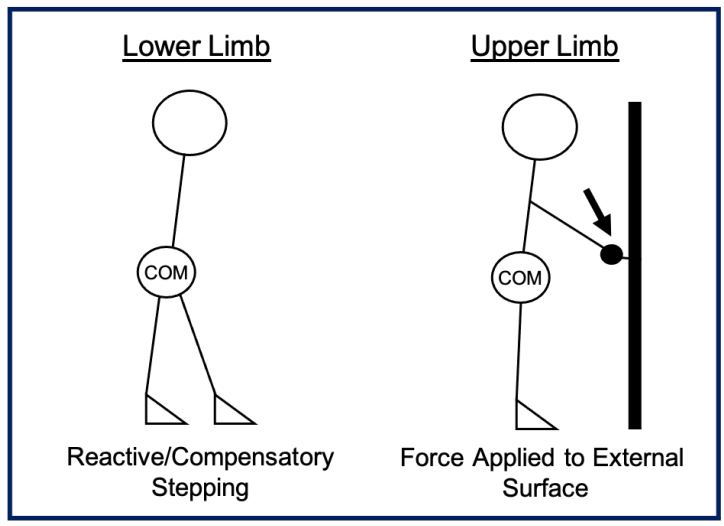

Figure 2.1 Balance recovery using fixed-support and change-in-support strategies (developed from Komisar et al. (2019) [27]).

\subsubsection{Change-in-Support Strategies: Change-in-support strategies are}

necessary to recover balance when a perturbation rapidly moves the COM beyond the limits of stability. Instead of pivoting at the ankle or hip joint to counteract COM movement, the size or shape of the BOS must be changed by moving a limb (arm or leg) to a different place of contact with the external environment to accommodate for the COM trajectory. This change in the dimensions of the BOS allows the COM to move farther away from the vertical position while still maintaining stability [24]. Change-insupport strategies can involve both compensatory stepping and grasping responses. Using 
the lower body, the BOS can be expanded by taking a compensatory step in the same direction that the COM is rapidly moving towards, in order to decelerate the COM [33]. In cases where a handrail or support surface is nearby, the upper limbs can act as moment arms by applying tactile feedback or forces to these surfaces which helps to decelerate the COM and provide stability [34].

\subsection{Factors Affecting Postural Control and Fall Risk}

Postural control is not based in a single neural system but is rather a complex integration of musculoskeletal (e.g., joint range of motion, muscle strength, biomechanical relationships of segments) and neurological (e.g., individual sensory systems, sensory organization, cognitive resources and strategies) components that affect balance ability and fall risk. Therefore, it is impossible to attribute falls to a single target factor [6]. Factors that can contribute to postural control (Figure 2.2) include biomechanical constraints (i.e., size of the BOS), movement strategies (i.e., reactive and anticipatory reactions), sensory strategies (i.e., sensory integration and reweighting), orientation in space (i.e., body position relative to gravity), control of dynamics (i.e., control of COM while changing postures), and cognitive processing (i.e., secondary cognitive tasks) [35]. If any of these components are impaired, postural stability decreases and fall risk increases. 


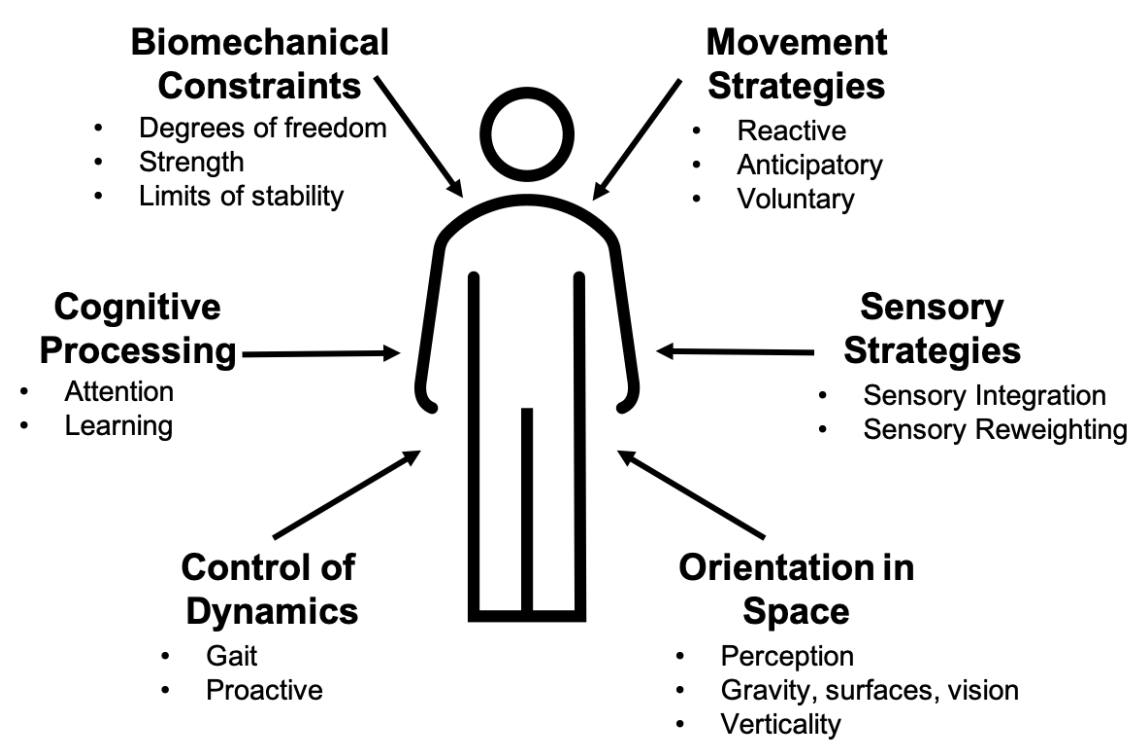

Figure 2.2 Factors contributing to postural control and fall risk (modified from Horak (2006) [35]).

\subsubsection{Investigating Fall Risk through Balance Recovery: Examining}

the effects of lighting on reactive balance can indicate how dark environments affect fall risk. Reactive balance can help indicate fall risk by providing a measure of stability and testing how effectively individuals can respond to instability [36]. Controlling the COM with respect to the BOS is crucial when preventing a fall. However, there is some evidence that COM control during standing balance is not a good indicator of balance recovery ability and fall risk [37]. On the other hand, many researchers have found certain measures during balance recovery to distinguish fallers from non-fallers and characterize those who can recover from a perturbation successfully from those who cannot. Generally, individuals who require fewer steps to recover their balance following a perturbation are more stable than individuals who take multiple steps. As stability decreases, particularly with age, multi-step responses are more often implemented [38] and necessary in response to smaller perturbations $[39,40]$. 
Given the need for a rapid motor response to prevent a fall following a perturbation, movement initiation and onset latencies of the involved musculature can also be indicative of fall risk. Following an induced laboratory slip, Sawers et al. (2017) found individuals who were not able to recover had longer onset latencies in knee flexors and extensors [41]. Delayed arm reactions can also increase the risk of falls and fall injury by inhibiting the ability to grasp handrails fast enough to protect the body from ground impact [42].

The effectiveness of balance recovery in preventing a fall has been attributed to many factors, involving both intrinsic and extrinsic factors [43]. These can be classified into three types of factors: 1) Individual (e.g., age, comorbidities), 2) Task (e.g., dual tasking, holding an object), and 3) Environmental (e.g., stair location, contact surface) [27] (Figure 2.3). This literature review was primarily focused on the influence of vision on balance control and balance recovery. 


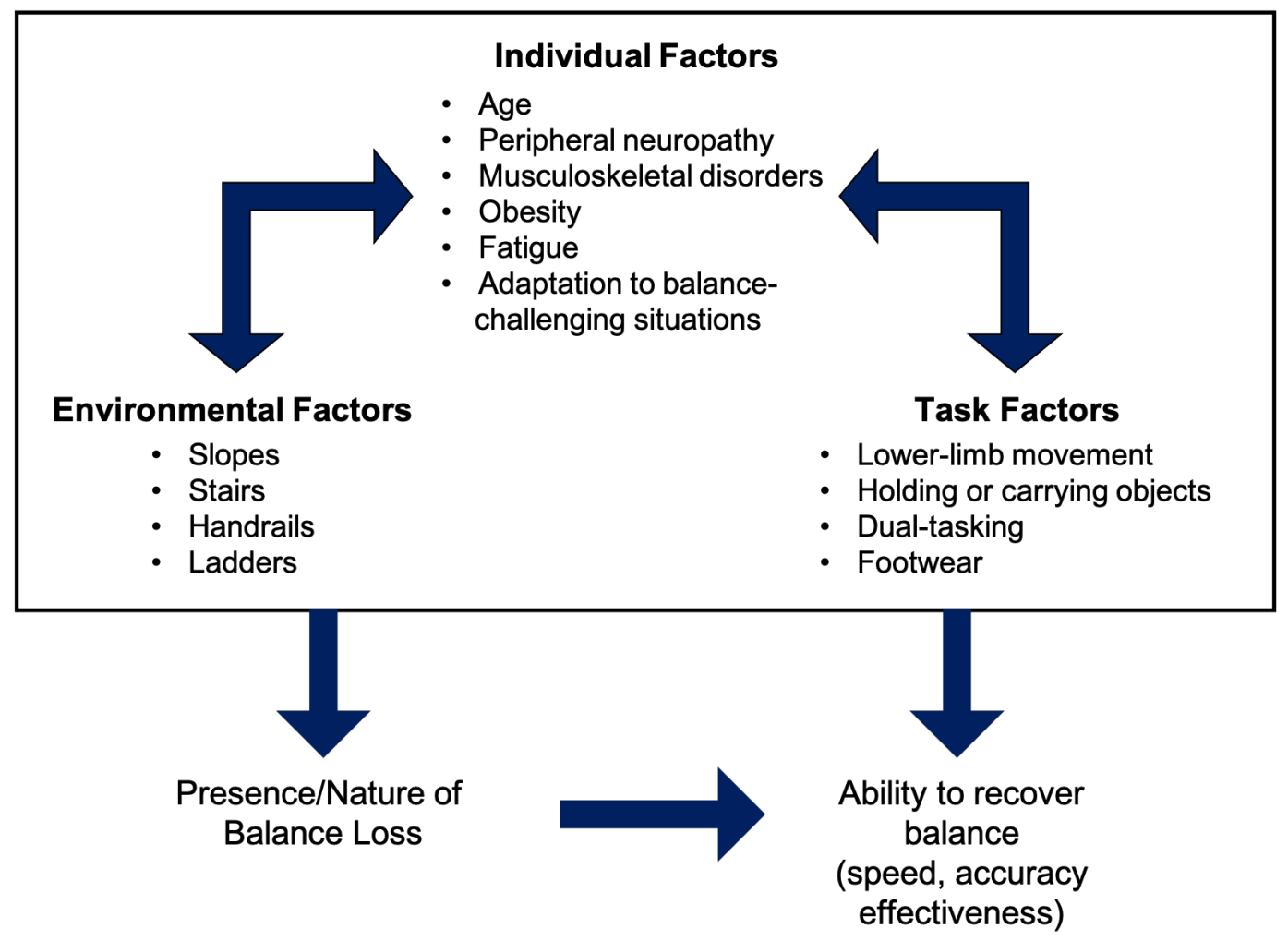

Figure 2.3 Individual, environmental, and task factors that influence balance recovery ability (developed from Komisar et al. (2019) [27]).

\subsection{Visual Contributions to Balance Control}

2.5.1 Vision and Anticipatory Balance: Dark environments could affect

balance recovery because they limit the availability of visual information. Vision is responsible for providing the balance control system with information about the external environment, and standing balance is affected when vision is challenged. During anticipatory balance, the visual system, particularly peripheral vision [44], senses changes in body orientation and can determine when the body is swaying and deviating from a stable posture by detecting changes in head and eye position [45]. Research generally suggests that when visual input is reduced or disoriented, postural sway increases, indicating that vision plays an important role in postural stabilization. 
When both young and old adults remove visual cues by closing their eyes, postural sway increases while standing [46] and sitting [47] due to reduced control over the COM. These results seem to indicate that when vision is removed, sensory reweighting to the somatosensory and vestibular systems cannot completely account for the loss of visual feedback, and postural stability is reduced. However, it appears that when vision is removed, providing additional sensory information to the somatosensory system can prevent this decrease in stability. Riley et al. (1997) found that when individuals stood with their index finger resting gently on a stable surface with their eyes closed, stability was equivalent to that with their eyes open [48]. Vennila and Aruin (2011) similarly found that individuals who held onto a walker while recovering from a perturbation with their eyes closed had comparable stability to individuals with their eyes open [49].

Older adults appear to be more affected than young adults when sensory information is impaired. Woollacott et al. (1986) found that young and older adults demonstrated similar amounts of body sway when only one sensory system (visual, vestibular, or somatosensory) was removed during a standing balance task. However, older adults experienced more body sway than younger adults when two sensory systems were removed [50]. Similarly, when older adults stood on a foam pad that challenged somatosensory input, sway was comparable to young adults, but body sway was much larger than young adults when their eyes were additionally closed [51]. However, these findings only examine postural control when visual cues are removed by closing the eyes or wearing a blindfold and do not examine alterations in visual information such as in dark environments. 
2.5.2 Vision and Gait: Many researchers have also examined the effect of reduced visual input on gait. While challenging the somatosensory, vestibular, or visual system, Chien et al. (2014) found that body sway during gait increased the most when visual input was removed, suggesting that vision is the primary sensory system providing postural feedback during gait [15]. Perry et al. (2001) found that when vision was covered using goggles, individuals had more variability in COM movements and delayed slowing of the forward movement of the COM when gait was terminated [52]. Individuals also have been found to walk with altered gait characteristics, including shorter stride length, limited hip movements, and a flatter foot during heel strike when vision was removed with a blindfold [53].

Some researchers have directly measured the effects of decreased lighting on gait, rather than removing visual cues by closing or covering the eyes. The primary outcome of decreased lighting on gait is a reduction in comfortable walking speed in both young [54] and older adults $[55,56]$. In individuals with gait disorders, decreased lighting also increases swing time, stride variability, and swing time variability [56]. Figueiro et al. (2011) found that when lighting was decreased while walking along a pathway, individuals were more unstable. However, providing a visual outline of the pathway in darkness increased stability and gait velocity in older adults with a high fall risk, suggesting that providing visual cues to define the walking environment can improve stability [55].

2.5.3 Vision and Reactive Balance: During reactive balance, vision is responsible for referencing body movements to the external world and mapping the 
environment to locate any support surfaces or obstacles to moving safely [5, 54].

Following a destabilization, the postural control system integrates sensory information regarding perturbation characteristics in order to implement the appropriate motor commands for regaining stability in a rapid and multi-segmented response. Research has shown that when the eyes are closed during support surface translation perturbations, head and trunk displacements increase $[9,57]$ and individuals resort to more of a hip strategy than ankle strategy to regain balance [10]. During relatively slow $(0.25-1.25 \mathrm{~Hz})$ instantaneous and continuous surface translations of $12 \mathrm{~cm}$, having eyes closed resulted in greater COM displacement and more head and trunk sway compared to with eyes open $[9,57]$. Similar findings were also found during rotational support surface translations (3$24 \mathrm{~cm} ; 0.08-3.67 \mathrm{~Hz}$ ), along with increased hip displacement and velocity relative to the ankle with the eyes closed, indicating more of a hip strategy than an ankle strategy to regain balance [10].

Decreased visual cues may also affect muscle activation and adaptation to repeated perturbations. During initial trials of balance perturbations, balance recovery is initially inappropriate and stiff, with large sway of the COM [58]. However, as more trials are completed, individuals adapt to muscle contraction patterns which are more effective and less energetically demanding [59]. Sozzi et al. (2016) examined lower leg muscular activations in response to sinusoidal platform displacements $(10 \mathrm{~cm} ; 0.6 \mathrm{~Hz})$ in individuals with eyes closed or eyes open. As more trials were completed, activation of the shank muscles decreased without affecting recovery success, indicating a more effective and less demanding response. This decrease in muscle activation occurred regardless of whether the trials were completed with eyes open or eyes closed, however, 
adaptation occurred twice as rapidly when vision was available. Individuals using visual information also demonstrated more coordination between body segments, smaller head displacements, and decreased co-contraction of the shank muscles as trials progressed [59].

These studies suggest that when closing the eyes and thereby removing the reference for body position relative to the external environment during reactive balance, stability and response adaptation are impaired. However, visual removal at the time of perturbation onset does not always reduce the ability to maneuver around obstacles when in a familiar environment. Awareness of environmental layout during balance recovery is crucial to avoid obstacles and uneven surfaces [54] and guide reaching motions to support surfaces [60], particularly when the response requires a stepping or reaching action. Many researchers have posed the question of whether this visuospatial information is accessed 'online', meaning processing occurs in real-time at perturbation onset, or whether a spatial map of the environment is formed and automatically updated as an individual encounters the environment [25].

The contribution of vision to balance recovery has been examined by temporarily removing vision either prior to or immediately after perturbation onset. Ghafouri et al. (2004) examined the role of timing of available visuospatial information on balance recovery when stepping responses were constrained (i.e., participants were forced to step in a particular manner to avoid obstacles). When vision was removed immediately after perturbation onset (i.e., relying on stored visuospatial information) individuals performed similarly to when vision was not removed. However, individuals with vision removed 
prior to the perturbation (i.e., no stored visuospatial information) displayed delays in stepping onset $(\sim 50 \mathrm{~ms})$ and contacted the barrier almost twice as frequently during stepping [61]. These results suggest that input latency of visuospatial information is too slow to effectively assist in mapping the environment during balance recovery, but stored visuospatial information can be used to guide balance recovery immediately following a perturbation and does not have to be processed at perturbation initiation.

\subsection{Environmental Lighting and Balance Control}

Dark environments can be experienced daily (e.g., getting out of bed at night, navigating through a movie theater, descending a dimly lit staircase), and pose a consistent threat to stability. Inadequate lighting can limit or distort visual information which provides the balance control system with a map of the environment and allows individuals to avoid obstacles, locate handrails, and plan movement routes [54]. Dim lighting is a risk factor for falling [8], however, there is limited information about how lighting affects balance mechanisms and the ability to prevent falls.

Addressing decreased lighting as a risk factor is beneficial because it is extrinsic and easily modifiable. Extrinsic risk factors (e.g., handrail design and environmental obstacles) can be modified in order to create safer physical environments for daily life when other intrinsic risk factors (e.g., age, comorbidities) cannot be changed [62]. Built environments have industry-recommended standards for lighting levels [63-65], however, ambient lighting conditions are often lower than recommended levels in independent living facilities [66] and personal homes [67, 68]. Inadequate lighting in personal, public, and occupational environments could lead to more falls. 
Instances of rapid transition between bright light and darkness could further increase fall risk. After exposure to bright illumination, the photopigment rhodopsin is bleached and rod photoreceptors are less sensitive [69]. Rhodopsin can take a few minutes up to almost an hour to regenerate, during which time rods are desensitized and visual stimuli are harder to detect $[70,71]$. Therefore, fall risk is greater when rapidly transitioning into a dark environment, such as switching off the lights before ascending stairs or getting into bed. While this idea has not been examined during balance recovery, Johnson and Meltzer (2012) found that after laying supine with eyes closed for 45 minutes, sway velocity was larger when standing up after pausing in a seated position for two seconds rather than for 30 seconds [72]. These results suggest that increasing adaptation times between different lighting conditions may help increase postural stability and reduce obstacle collision.

It should also be considered that aging may affect how individuals respond to dark environments. Older adults are already at a greater risk for falls and fall injury than young adults [73], but also experience age-related declines in vision including impaired spatial contrast sensitivity [74], decreased lens transmittance capacity [71], and slower visual processing speeds [75]. These declines may affect the reliability of visual information when lighting is decreased. Older adults also experience substantial delays in dark adaptation [76] due to a delayed rhodopsin regeneration process [70] and a lower density of rod photoreceptors [77]. This may affect how quickly they regain contrast sensitivity when transitioning into a dark environment. 


\subsection{Remaining Questions from the Literature}

Research indicates that when visual input is limited, stability is reduced during both anticipatory and reactive balance. However, a majority of the previously mentioned studies removed visual cues by closing or covering the eyes and did not test balance in actual darkness. Hafström et al. (2002) suggest that postural responses are not the same in a dark environment as they are with the eyes closed. During a standing balance task, individuals demonstrate larger initial COM sway with the eyes open in a dark environment than with the eyes closed in a dark environment, which may be due to delayed sensory reweighting when visual information is expected with the eyes open [78]. Additionally, a dark room could still have some sources of light that prevent complete visual loss and provide more visual information than is available when blindfolded or with the eyes closed. Rugelj et al. (2014) found that postural sway was larger in complete darkness than in low illumination, indicating that even low levels of lighting can help improve balance compared to complete darkness [79]. Therefore, postural responses in dark environments may not be the same as when the eyes are closed or covered. The effects of dark environments should be considered given that individuals are more likely to experience instances of darkness than instances where the eyes are closed or covered in real-world situations.

Additionally, a majority of previous research has examined the effects of visual cue removal on fixed-support balance strategies (i.e., feet-in-place strategies). These studies elicited relatively small perturbations that did not require a change in the BOS to recover balance. However, most individuals take steps to recover balance following real-world 
slips and trips [33]. It is unknown how dark environments will affect reactive stepping responses when visual information is not available.

Examining how dark environments affect reactive balance is critical to understand how individuals are able to prevent falls in different lighting conditions. This is particularly important because dim lighting can decrease the visibility of tripping hazards and may increase losses of balance. This thesis examined how balance mechanisms are different during reactive stepping in a well-lit and dark environment. This research is a crucial foundational step to understand fall risk and prevention in different conditions of ambient lighting and will contribute to information about designing, assessing, and modifying built environments to improve safety. 


\section{Research Study Overview}

\subsection{Purpose}

The overall purpose of this research study was to examine the effects of decreased ambient lighting on reactive balance mechanisms. To reduce falls in dark environments, it must be understood how decreased lighting affects the ability to recover from losses of balance and prevent falls. Ultimately, this research provides knowledge about the importance of lighting conditions when designing, assessing, and modifying environments to increase safety. It also provides objective information to clinicians to allow the development of balance training interventions that minimize fall risk.

\subsection{Delineation of Papers}

I conducted one research study which I present as two papers to answer different research questions. Paper 1 analyzed the effects of decreased ambient lighting on the success of balance recovery responses, reactive stepping kinematics, and COM control following forward losses of balance to examine how body movements and stability are modified when recovering balance in dark environments. Paper 2 analyzed the activation, timing, and coordination of lower-limb muscles during the same experimental protocol to examine how dark environments can affect neuromuscular responses while anticipating, detecting, and responding to perturbations. The same participants were included in both papers, with the exception of one participant who could not be included in the analysis of neuromuscular data (Paper 2). 


\subsection{Hypotheses}

Overall, I hypothesized that decreased lighting would affect reactive balance mechanisms and the ability to recover balance. I hypothesized that decreased lighting would impair the ability to regain balance, as indicated by decreased maximum recoverable perturbation magnitude (maximum lean angle) and decreased COM control. Additionally, I hypothesized that decreased lighting would affect reactive stepping trajectories, resulting in changes to stepping parameters and lower-limb angular kinematics. With respect to neuromuscular responses, I hypothesized that decreased lighting would result in an overcompensated and less coordinated lower-limb muscular response, including greater activation amplitudes and stiffening between muscles, but no change in response initiation time. Specific outcomes and hypotheses for each paper are outlined in Table 3.1 . 
Table 3.1 Breakdown of Thesis Papers

\begin{tabular}{|c|c|c|}
\hline & $\begin{array}{l}\text { Paper 1: Reactive Stepping } \\
\text { Responses and COM Control }\end{array}$ & $\begin{array}{l}\text { Paper 2: Lower-Limb } \\
\text { Neuromuscular Responses }\end{array}$ \\
\hline Participants & 20 young adults & 19 young adults \\
\hline Outcomes & $\begin{array}{l}\text { - } \text { Stepping Parameters } \\
\text { - } \text { Angular Kinematics } \\
\text { - } \text { COM Kinematics } \\
\text { - Postural Stability } \\
\text { - } \text { Maximum Lean Angle }\end{array}$ & $\begin{array}{l}\text { - Mean and Maximum Muscle } \\
\text { Activation Amplitudes } \\
\text { - Muscle Onset Latencies } \\
\text { - Co-Contraction Index } \\
\text { - Cross-Correlational Coefficients }\end{array}$ \\
\hline \multirow[t]{2}{*}{ Hypotheses } & $\begin{array}{l}\text { H1: Decreased lighting will impair the } \\
\text { ability to regain balance } \\
\text { - } \quad \downarrow \text { maximum lean angle } \\
\text { - } \quad \downarrow \text { postural stability } \\
\text { - } \uparrow \text { COM kinematics }\end{array}$ & $\begin{array}{l}\text { H1: Decreased lighting will result in } \\
\text { an overcompensated muscular } \\
\text { response } \\
\text { - } \uparrow \text { muscle activation amplitudes } \\
\text { - } \uparrow \text { stiffening/co-contraction } \\
\text { - } \quad \text { coordination between muscles } \\
\text { (cross-correlation coefficients) }\end{array}$ \\
\hline & $\begin{array}{l}\text { H2: Decreased lighting will modify } \\
\text { stepping responses } \\
\cdot \quad \text { Altered stepping parameters and } \\
\text { angular kinematics }\end{array}$ & $\begin{array}{l}\mathrm{H} 2 \text { : Decreased lighting will not affect } \\
\text { response initiation time } \\
\text { - No difference in muscle onset } \\
\text { latencies }\end{array}$ \\
\hline
\end{tabular}




\section{Paper 1 - The effect of decreased ambient lighting on reactive stepping kinematics and center of mass control following forward losses of balance}

\subsection{Introduction}

Falls occur in adults of all ages and result in substantial injuries, hospitalizations, and healthcare costs [2]. There are many extrinsic risk factors for falls including slippery surfaces, absence of handrails, and stair dimensions [80]. Dim or poor lighting is another risk factor for falling $[8,81]$ and is experienced daily by most individuals in home, work, and public environments. Although post-fall assessments have found that many falls occur in dark environments $[11,12]$, the relationship between dark environments and balance control has not been as widely researched as other fall risk factors (e.g., age, comorbidities). Specifically, it is unknown how balance control mechanisms are affected by dark environments.

Postural control requires integration of vestibular, somatosensory, and visual systems to detect deviations from a stable posture and implement corrective motor responses [5]. The central nervous system (CNS) 'weights' the input from each sensory system based on the proportional availability of sensory information, and can further adjust sensory dependence when environmental constraints alter sensory information [20]. The visual system is responsible for detecting changes in head and eye position relative to the external environment and mapping the environment to locate obstacles, handrails, stairs, etc. [45]. Dark environments challenge balance control and may contribute to falling because less visual information is available.

The material contained in Chapter 4 is in preparation for submission to Applied Ergonomics. 
To date, most research examining decreased vision has focused on balance during quiet stance $[46,82,83]$. When visual input is decreased or disoriented, postural sway generally increases, indicating that vision plays an important role in postural stabilization. Reactive balance mechanisms used in response to losses of balance differ from voluntary movements by the speed at which environmental cues regarding destabilization must be integrated, requiring a more rapid response in order to prevent falls $[24,27,33]$. The few studies that have investigated reactive balance in instances of decreased vision have primarily focused on relatively small, cyclic, and instantaneous support surface translations of short duration that do not require stepping to maintain balance (i.e., feetin-place/fixed-support reactions) $[9,10,84]$. When vision is decreased during these types of support surface translations, individuals show increased head, trunk, and center of mass (COM) displacement [9] and shift from an ankle to a hip strategy to regain balance [10]. However, these response modulations did not always affect overall balance recovery ability [84]. While the existing literature provides an understanding of the effects of decreased vision on fixed-support strategies, it is still unknown how decreased vision will affect responses when reactive stepping is required to accommodate the movement of the COM [33].

Furthermore, while the role of vision during postural control has been examined via visual cue removal methods (e.g., blacked out goggles, blindfold, eyes closed), little is known about the effects of decreased ambient lighting on balance control, and whether dark environments will have different effects than visual cue removal. Hafström et al. (2002) suggest that the brain expects to receive visual information when the eyes are open in a dark environment, and delays reweighting sensory dependence to the vestibular 
and somatosensory systems [78]. Therefore, sensory reweighting and the resulting reactive balance mechanisms in dark environments where the eyes remain open may differ from postural control when the eyes are closed or vision is occluded with a blindfold.

In order to reduce the number of falls experienced due to dim lighting, it is important to understand how reactive balance control is affected in dark environments. Research in this area is needed to establish standard levels of light for optimal balance control in work and public environments. Although current lighting standards exist [63, 64], they are not based on preventing falls or balance control, and lighting levels in independent living facilities [66] and personal homes [67] are still typically lower than industry-recommended standards. Understanding how changes in lighting conditions affect the ability to detect postural instabilities and develop effective balance recovery responses is essential to further our understanding of sensory reweighting and strategy development to prevent falls in areas of limited lighting.

Our study examined how decreased lighting affected reactive balance mechanisms in response to 'lean-and-release' perturbations. Specifically, we examined the effects of complete darkness on maximum lean angle, stepping kinematics (step length, width, height, velocity, time, and onset time), angular kinematics (relative velocities of the foot and shank (ankle); shank and thigh (knee), thigh and pelvis (hip); pelvis and thorax (trunk)), COM kinematics (displacement, velocity, and acceleration), and postural stability (COM movement relative to the base of support (BOS)) during reactive balance mechanisms to known lean-and-release perturbations in healthy, young adults. We 
hypothesized that decreased lighting would reduce the ability to successfully regain balance, resulting in a lower maximum lean angle at which successful balance recovery was possible and decreased postural stability (decreased $\mathrm{X}_{\mathrm{COM} / \mathrm{Bos}}$ values). We also hypothesized that decreased lighting would result in altered stepping and angular kinematics and increased COM displacements, which may indicate lower levels of stability at a given lean angle.

\subsection{Methods}

4.2.1 Participants: 20 young adults between the ages of 18-30 years (mean (SD); age: 23.3(4.4) y; 10 female, 10 male; mass: 74.7(17.2) kg; height: 1.7(0.1) m) were recruited to participate in the study. All participants were free of osteoporosis, injuries to the neck, head, trunk, and lower body, vertebral body compression fractures, traumatic brain injuries, and vestibular/neurological disorders that might affect balance.

Participants were instructed to wear correctional lenses if they were normally worn during daily living. Prior to data collection, all participants provided written consent, confirmation of participant eligibility (Appendix A), and a brief medical history (Appendix B). This study was approved by the institution's Institutional Review Board $(1433509-2)$

\subsubsection{Study Protocol: A custom-built lean-and-release system was used to} induce forward losses of balance (Figure 4.1). Participants wore a fall arrest harness connected to a free-standing gantry. The harness was attached to a tether which connected to the wall posterior to the participant, allowing the participant to lean forward without losing their balance. Participants were asked to lean forward by pivoting at the 
ankle while maintaining a plank-like position in the hips and upper body to prevent any bending at the hip. The length of the tether was adjusted to change the lean angle of the participant (measured via goniometer from the lateral malleolus). Participants stood in this position with the heels $17 \mathrm{~cm}$ apart and a $14^{\circ}$ angle between the feet [85]. The tether was then released at a random time interval between $0-30$ seconds after the participant leaned forward in the harness, causing the participant to fall forward. The participant was instructed to minimize the number of steps taken to recover their balance.
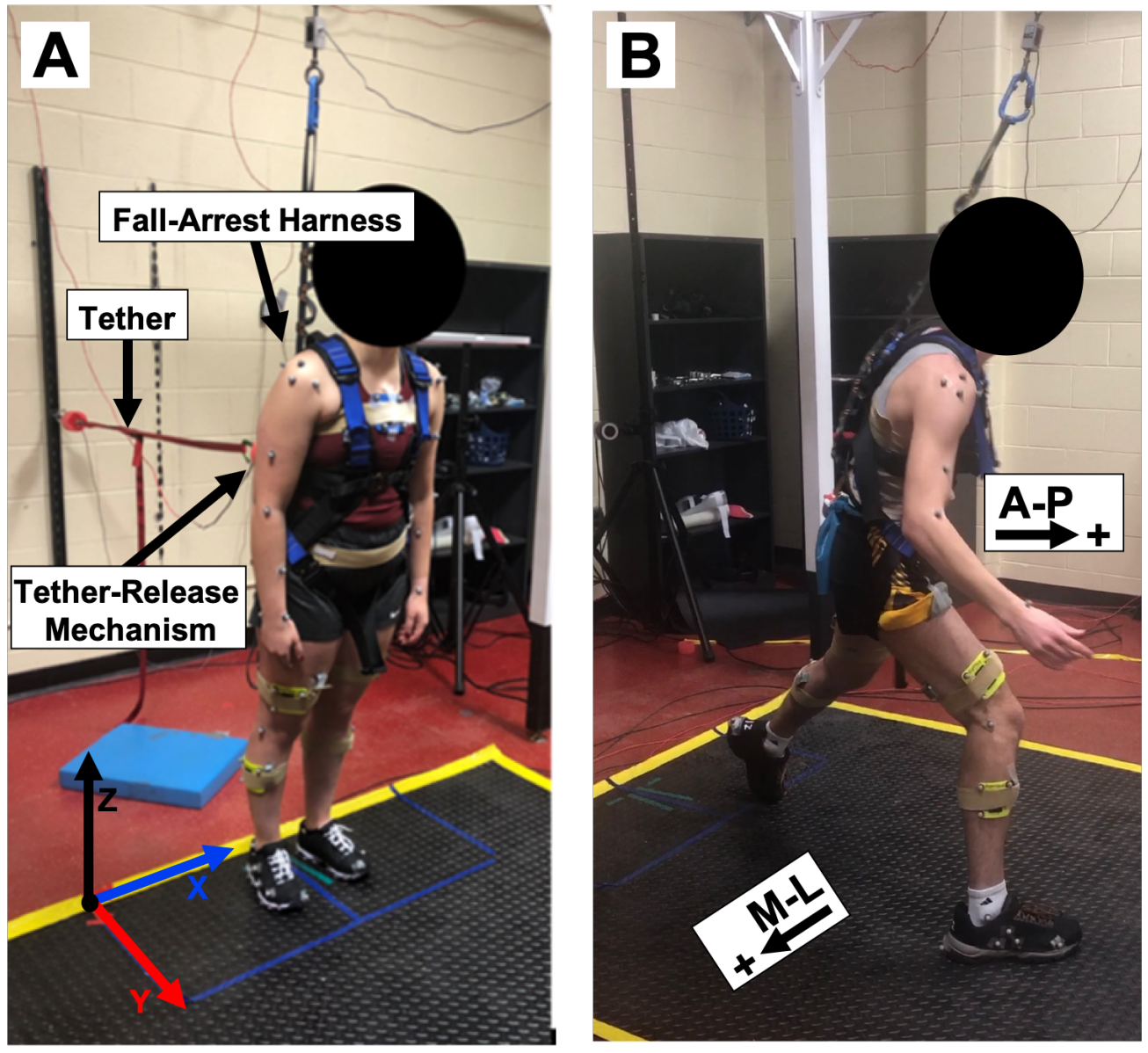

Figure 4.1 (A) Lean-and-Release Fall Arrest System with coordinate system. (B) Image to interpret (+/-) values in anterior-posterior (A-P) and medial-lateral (M-L) directions.

$(+)$ values in the A-P direction indicate forward movement. $(+)$ values in the M-L direction indicate movement toward the stepping leg. 
Participants completed 12 practice trials at a $10^{\circ}$ lean angle to minimize the effects of habituation on trials used to test the study hypotheses [86], while consistently eliciting single step responses [87]. The participants then completed a ramp-up protocol to maximum single step lean angle [88] in 2 lighting conditions: 1) Light and 2) Dark. In the Light condition, the testing space was illuminated by standard overhead fluorescent lights ( $\sim 800$ Lux). In the Dark condition, all lights were turned off (0 Lux). To limit the effects of dark adaptation, the lights were turned off no more than $30 \mathrm{~s}$ before the perturbation, and the testing space was normally illuminated by overhead lights in between each trial [69]. In each lighting condition, participants began trials at a $10^{\circ}$ lean angle. The lean angle was subsequently increased by $2^{\circ}$ each trial until the participant failed to recover their balance. A balance failure was classified by taking more than 1 step or taking 1 step forward but letting the back midfoot move from the original position. When a participant failed to recover their balance at a given angle, the same angle was repeated. If a participant failed to recover their balance in 3 of 5 attempts, it was considered their maximum lean angle and that lighting condition was completed. If the participant could successfully recover their balance in 3 of 5 attempts, the ramp-up protocol continued until maximum lean angle was reached. Participants were given time to rest if needed in between each trial. The order of conditions was randomized to limit the effects of fatigue and learning.

4.2.3 Data Acquisition: Body movements were captured using an 11 camera Qualisys 3D motion capture system (Qualisys Inc., Sweden). A full-body 74-marker set was used to define joint locations and segment lengths, and motion trials were completed 
with a 64-marker set to track segment movements. Individual markers were placed on the head (4), shoulders ( 4 per side), arms (6-8 per side), legs ( 4 per side), and feet (6 per side). Four markers were also placed on the tether at the point of release to determine tether release time. Segment tracking markers were mounted on rigid clusters (3-4 markers per cluster) and placed on the thorax, pelvis, thighs, and shanks. COM and movement kinematics were calculated using a 13-segment (head, thorax, upper arms, forearms, pelvis, thighs, shanks, and feet) kinematic model developed in Visual 3D v.6 (C-Motion Inc., USA).

\subsubsection{Data Analysis:}

Motion Capture Signal Processing and Event Onset. Marker position data were low-pass filtered with a fourth-order, zero-lag Butterworth filter (cutoff frequency: $6 \mathrm{~Hz}$ ) [89]. Stepping parameters were calculated during the first step of the stepping reaction, defined as toe-off to subsequent heel-strike of the same foot. Toe-off was defined as the peak positive velocity of the foot COM in the vertical direction. Heel-strike was defined by the second peak negative velocity of the foot COM in the vertical direction, or as the maximum peak negative velocity if only 1 peak was present $[90,91]$. All toe-off and heel-strike events were examined for correctness and manually altered if the events did not occur at these peak velocities. Tether release time was determined by the initial acceleration of tether markers (i.e., when acceleration deviated from $0 \mathrm{~m} / \mathrm{s}^{2}$ ). All outcomes were calculated for the lean angle at which each participant could successfully recover their balance with 1 step in both Light and Dark conditions (Appendix C). Due to the design of the ramp-up protocol, participants completed only 1 trial at this lean angle in each condition. 
Stepping Parameters. Step length was calculated as the anterior-posterior (A-P) distance between the heel marker of the stepping foot in the forward lean position before tether release and the heel marker at heel-strike. Step length was normalized as a percentage of body height. Step height was defined as the highest point of the heel marker on the stepping foot in the vertical direction between toe-off and heel-strike. Step width was calculated as the medial-lateral (M-L) distance between the heel marker on the standing foot in the forward lean position before tether release and the heel marker on the stepping foot at heel-strike. Step onset time and step time were defined as the times between tether release and toe-off and between toe-off and heel-strike, respectively. Step velocity was calculated as the peak velocity of the COM of the stepping foot segment in the A-P direction between toe-off and heel-strike.

Angular Kinematics. We calculated peak positive and negative joint velocities between tether-release and heel-strike at the ankle, knee, hip, and trunk rotating about the $\mathrm{x}$-axis, with respect to the vertical direction (coordinate system indicated in Figure 4.1). The relative movement of the following segments of the stepping limb were used to calculate angular velocities: foot and shank (ankle); shank and thigh (knee); thigh and pelvis (hip); pelvis and thorax (trunk). Positive values represent ankle dorsiflexion, knee flexion, hip flexion, and trunk flexion.

COM Kinematics. COM location was estimated in Visual 3D as a weighted average of each body segment's approximate COM [92]. COM displacement was calculated by subtracting COM position at heel-strike from COM position at tetherrelease in the A-P and M-L directions. Velocity and acceleration of the COM were 
calculated by taking the first and second derivatives of the COM position, respectively. Maximum COM velocity and acceleration in the A-P and M-L directions were calculated between tether release and heel-strike.

Postural Stability. Postural stability was calculated at heel-strike in the A-P and M-L directions. Postural stability was quantified with the measure $\mathrm{X}_{\mathrm{COM} / \mathrm{BOs}}$, which calculates the weighted sum of the position and velocity of the COM relative to the position and velocity of the BOS $[93,94]$. This measure has previously been used to assess postural stability during reactive slipping [94, 95] and tripping [96]. The position and velocity of the COM was subtracted from the position and velocity of the BOS to

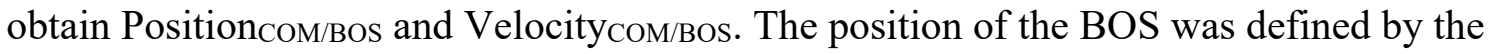
anterior or lateral border of the stepping foot, determined by the metatarsal markers [96]. Postural stability $\left(\mathrm{X}_{\mathrm{COM} / \mathrm{BOs}}\right.$ ) was calculated using Equation 4.1 , where ' $\mathrm{g}$ ' is equal to the gravity constant $\left(9.81 \mathrm{~m} / \mathrm{s}^{2}\right)$ and ' $l$ ' represents leg length (distance between lateral malleolus marker and estimated head of femur).

Equation 4.1: $\mathrm{X}_{\mathrm{COM} / \mathrm{BOS}}=$ Position $_{\mathrm{COM} / \mathrm{BOS}}+\frac{\text { velocity }_{\mathrm{COM} / \mathrm{Bos}}}{\sqrt{g / l}}$

Statistical Analysis. Differences in maximum lean angle, stepping parameters, and angular kinematics were compared between lighting conditions (Light, Dark) using paired sample t-tests. COM kinematics and $\mathrm{X}_{\mathrm{COM} / \mathrm{BOs}}$ were also compared between lighting conditions (Light, Dark) using paired sample t-tests for each direction (A-P, M-L). The differences in dependent variables between lighting conditions were normally distributed, meeting the assumptions for paired sample t-tests. Cohen's D was used to calculate the effect size of Lighting. Hotilly's $\mathrm{T}^{2}$ test was also used to analyze the effect 
of lighting on multiple variables at once, including all stepping parameters, angular kinematics, and measures of COM control (COM displacement, velocity, acceleration, and postural control). Pearson correlations were used to analyze the relationships between variables that were significantly affected by lighting, by examining correlations between changes in each variable from Light to Dark. Statistical analyses were performed using SPSS v. 26 (IBM Corp, SPSS Statistics, USA). An alpha of 0.05 was used for all tests.

\subsection{Results}

4.3.1 Maximum Lean Angle: Maximum lean angle ranged from $16^{\circ}$ to $44^{\circ}$

(Light:16-44우 ${ }^{\circ}$ Dark: $20-42^{\circ}$ ) and did not significantly differ between lighting conditions $(p=0.32)$. Lean angle data can be found in Appendix C.

4.3.2 Stepping Parameters: Multivariate testing including all stepping parameters originally indicated no effect of lighting $\left(T^{2}(6,33)=0.846, p=0.95\right)$. Paired sample t-tests for each stepping parameter indicated that lighting condition significantly affected step length $(\mathrm{t}(19)=-2.26, \mathrm{p}=0.04)$. Step length was longer in Dark $(56(2) \%$ of height) than in Light (53(2)\%). Lighting condition did not affect step width (21(4) cm; $\mathrm{p}=0.54)$, height $(28(4) \mathrm{cm} ; \mathrm{p}=0.34)$, velocity $(6.22(1.03) \mathrm{m} / \mathrm{s} ; \mathrm{p}=0.18)$, time $(190(23) \mathrm{ms}$; $\mathrm{p}=0.19)$, or onset time $(270(34) \mathrm{ms} ; \mathrm{p}=0.35)$ (Figure 4.2) Paired sample t-test results and effect size for all variables can be found in Appendix D. 

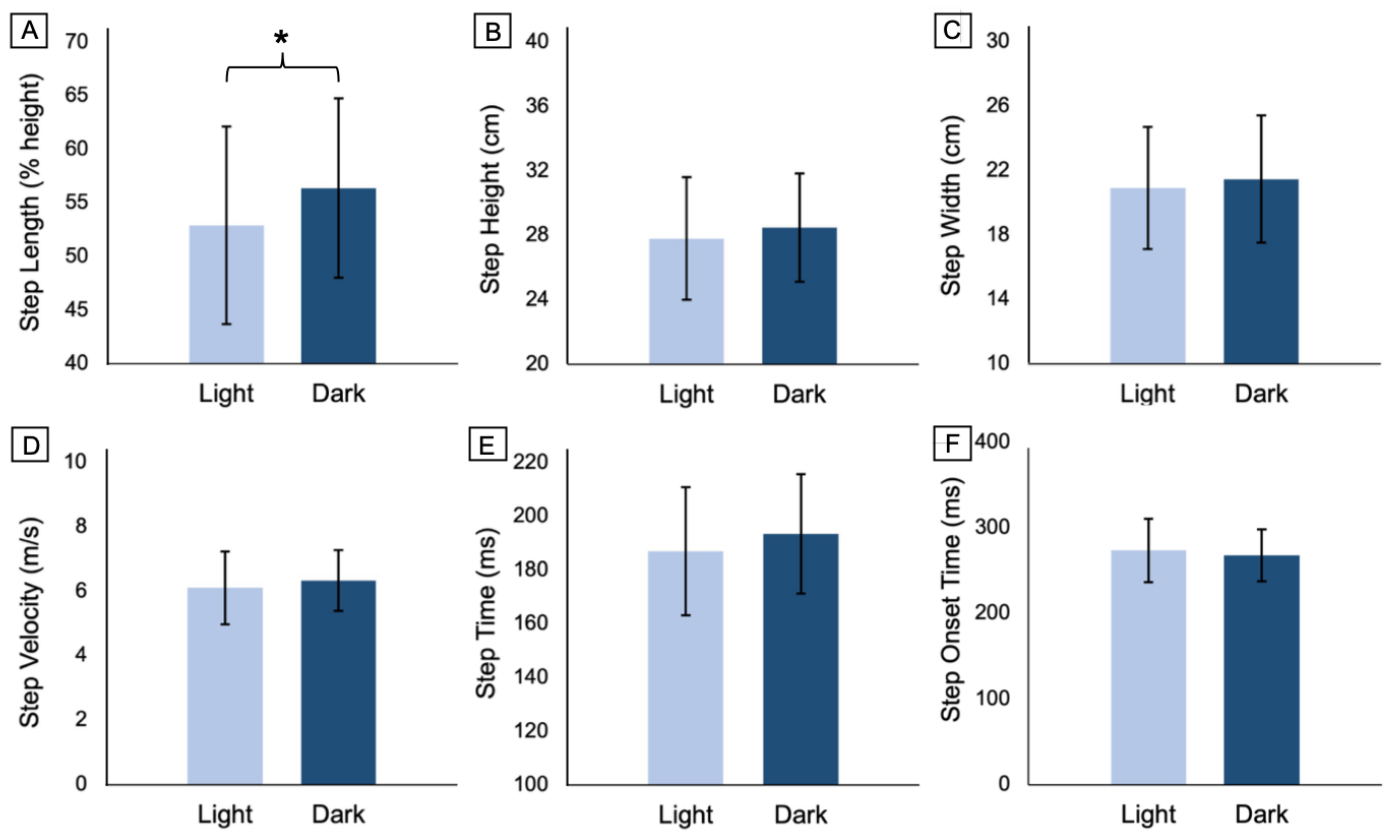

Figure 4.2 Step length (A), height (B), width (C), velocity (D), time (E), and onset time $(\mathrm{F})$ in different lighting conditions; Mean $\pm \mathrm{SD}$. * significant difference $(\mathrm{p}<0.05)$ between lighting conditions.

4.3.3 Angular Kinematics: Multivariate testing including all joints indicated no overall effect of lighting $\left(\mathrm{T}^{2}(8,24)=2.73, \mathrm{p}=0.78\right)$. However, individual $\mathrm{t}$-tests indicated that lighting condition significantly affected angular velocity at the knee $(\mathrm{p}=0.02)$, hip $(\mathrm{p}=0.05)$, and trunk $(\mathrm{p}<0.01)$. Knee flexion and extension had higher peak velocities in Dark than Light (Flexion: 6\% increase; Light: 524(16)\% $/ \mathrm{s}$; Dark: 558(18)\% $/ \mathrm{s})$; Extension: 4\% increase; Light: $-618(90)^{\circ} / \mathrm{s}$; Dark: $\left.-645(60)^{\circ} / \mathrm{s}\right)$. Peak hip flexion and trunk extension velocity were also higher in Dark than Light (Hip: 9\% increase; Light: 419(23)\%/s; Dark: 455(26)\%/s; Trunk: 28\% increase; Light: $-152(87)^{\circ} /$ s; Dark: $\left.-195(86)^{\circ} / \mathrm{s}\right)$. Lighting condition did not have a significant effect on ankle dorsiflexion $\left(87(47)^{\circ} / \mathrm{s} ; \mathrm{p}=0.43\right)$ or plantarflexion $\left(-317(82)^{\circ} / \mathrm{s} ; \mathrm{p}=0.37\right)$ velocity (Figure 4.3$)$. Individual changes in angular velocities were not significantly correlated with changes in step length $(\mathrm{p}>0.05)$. 


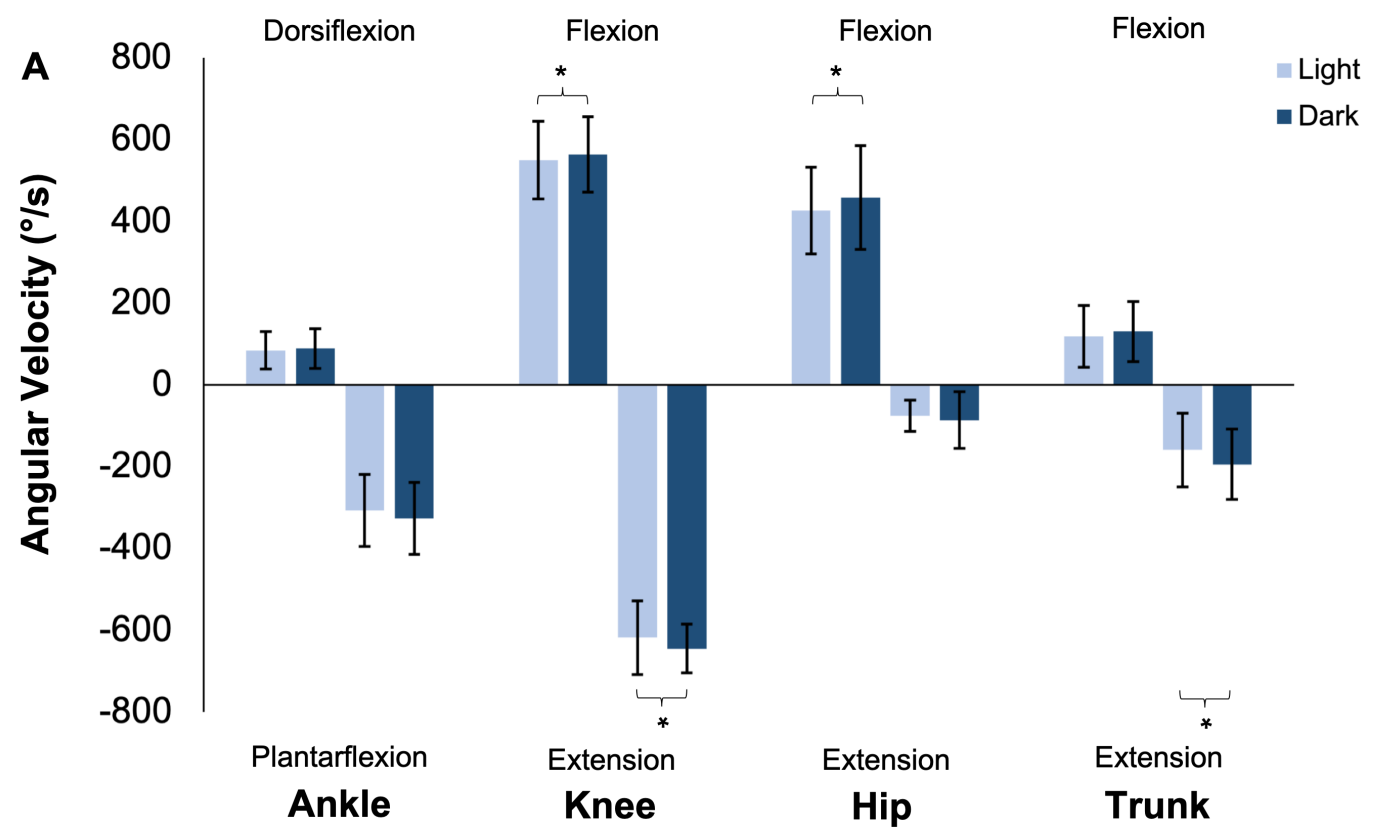

B
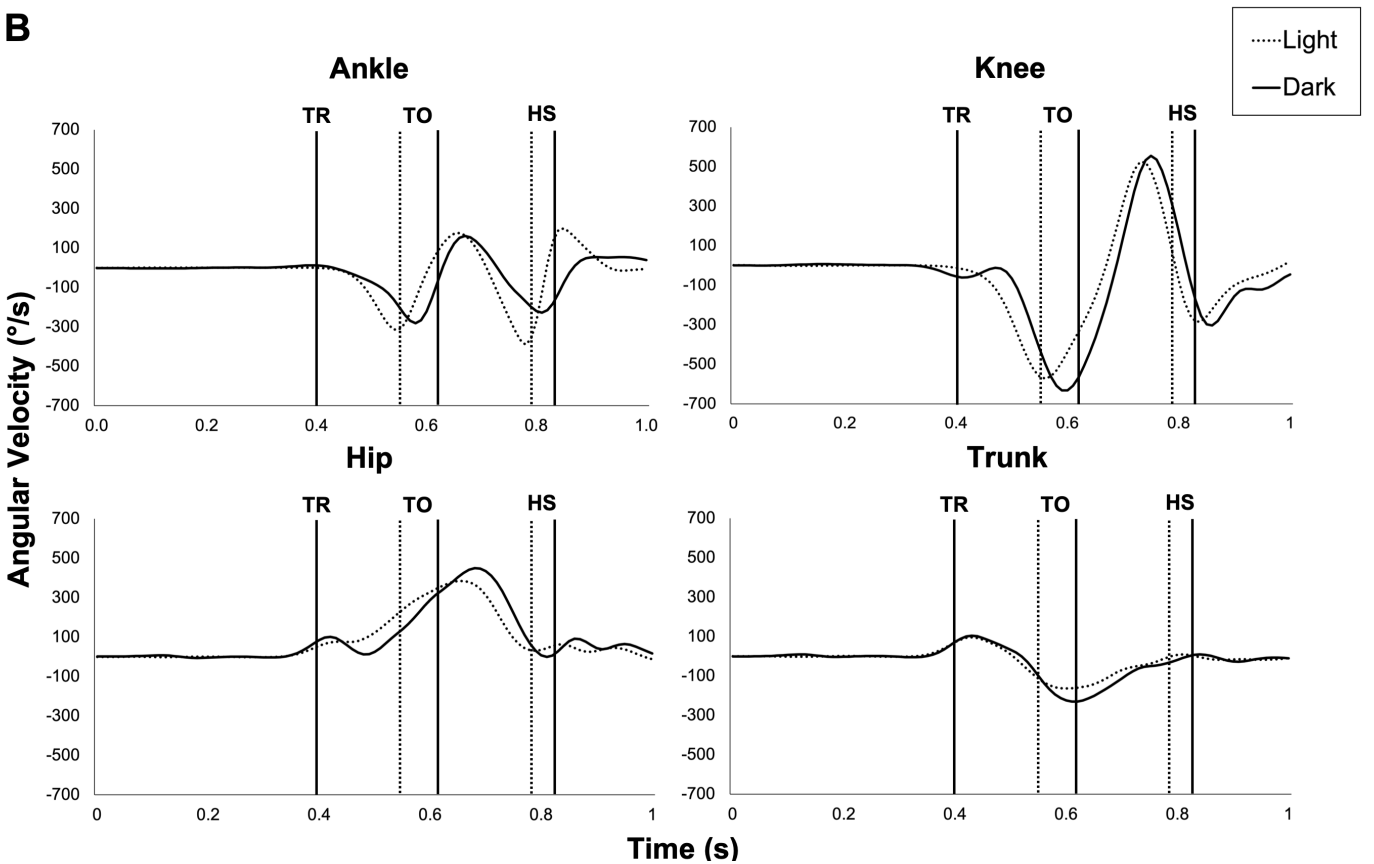

Figure 4.3 (A) Peak +/- angular velocity at the ankle, knee, hip, and trunk; Mean \pm SD. *significant difference $(\mathrm{p}<0.05)$ between lighting conditions. (B) Angular velocity at the ankle, knee, hip, and trunk during initial reactive step in representative participant. TR: tether release; TO: toe-off; HS: heel-strike. 
4.3.4 COM Movement: Multivariate testing including COM displacement,

velocity, and acceleration, and postural stability indicated no overall effect of lighting on COM control $\left(\mathrm{T}^{2}(8,31)=7.13, \mathrm{p}=0.19\right)$. Individual $\mathrm{t}$-tests indicated that lighting condition did not have a main effect on COM displacement (A-P: 0.03(0.01) m; M-L: 0.30(0.06)

m), peak velocity (A-P: $0.18(0.04) \mathrm{m} / \mathrm{s})$; M-L: $1.20(0.21) \mathrm{m} / \mathrm{s})$, or peak acceleration (A-P: $\left.1.65(0.41) \mathrm{m} / \mathrm{s}^{2} ; \mathrm{M}-\mathrm{L}: 5.55(1.27) \mathrm{m} / \mathrm{s}^{2}\right)(\mathrm{p}>0.05)$ (Figure 4.4).

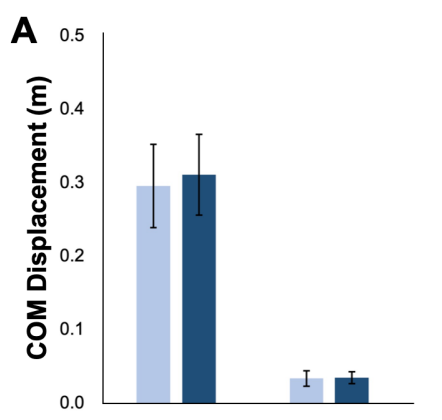

B
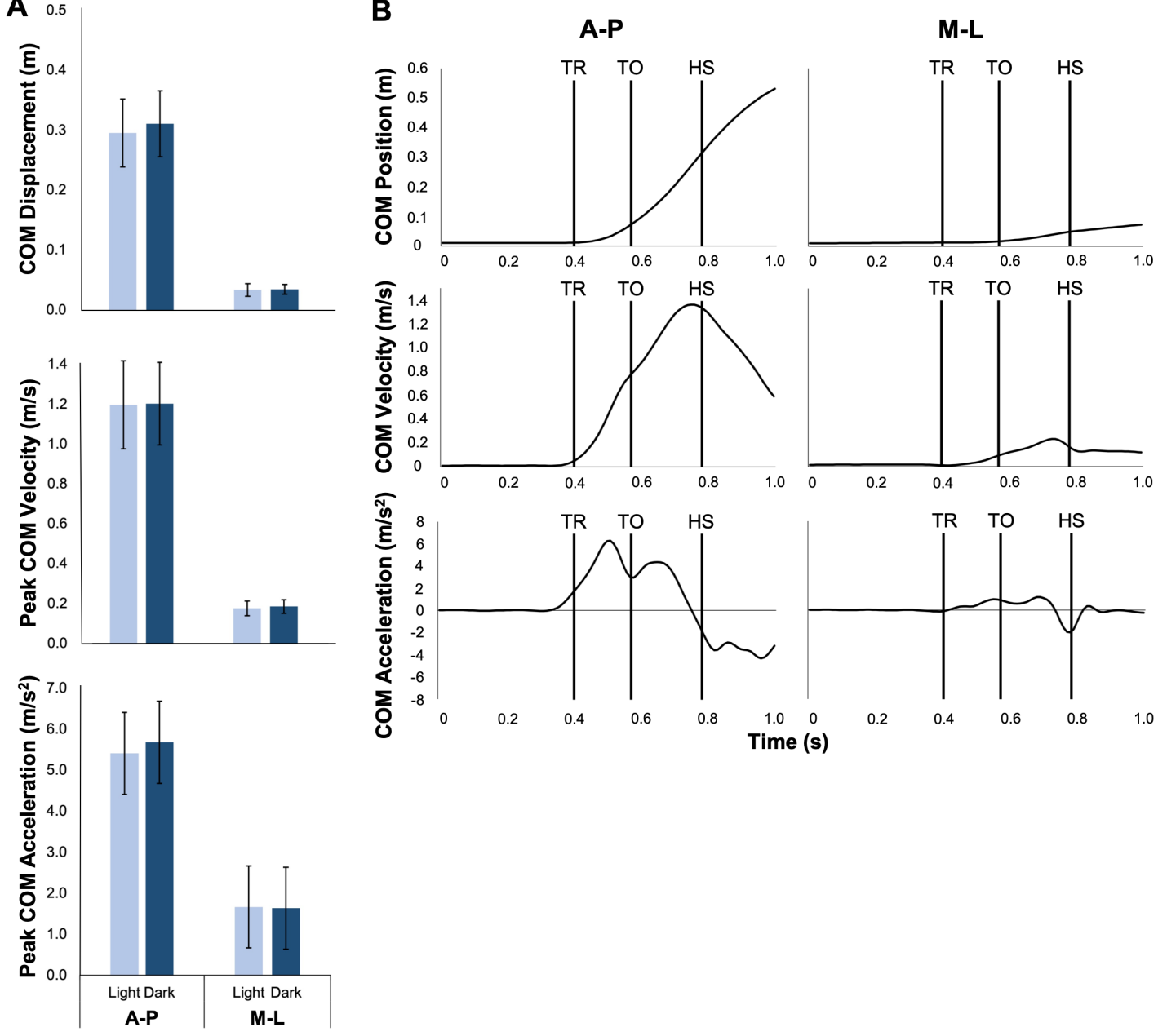

Figure 4.4 (A) Peak COM displacement, velocity, and acceleration; Mean \pm SD.

*significant difference $(\mathrm{p}<0.05)$ between lighting conditions. (B) COM position (relative to initial lean position), velocity, and acceleration during initial reactive step in single participant (Light Condition). TR: tether release; TO: toe-off; HS: heel-strike. 
4.3.5 Postural Stability: Individual t-tests indicated that lighting affected $\mathrm{X}_{\mathrm{COM} / \mathrm{Bos}}$ in the A-P direction $(\mathrm{t}(19)=2.19, \mathrm{p}=0.04)($ Figure 4.5$) . \mathrm{X}_{\mathrm{COM} / \mathrm{Bos}}$ was larger in Light (70(26)\% of step length) than in Dark (58(18)\% of step length). However, both values indicate high levels of stability. Lighting condition did not affect M-L postural stability (31(27)\% of step width; $\mathrm{p}=0.34)$. Changes in A-P postural stability between lighting conditions were not significantly correlated with changes in angular velocities or step length $(\mathrm{p}>0.05)$.

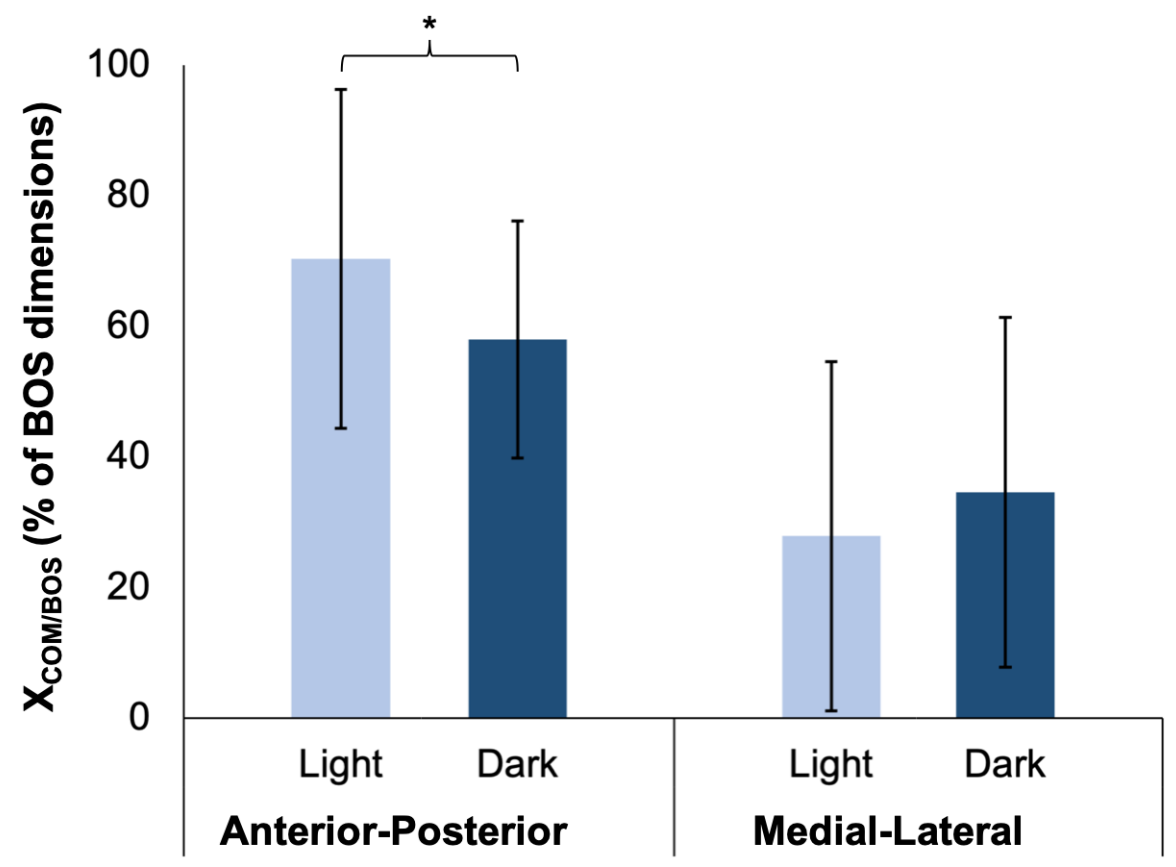

Figure 4.5 $\mathrm{X}_{\mathrm{COM} / \mathrm{BOS}}$ values in lighting conditions presented as a percentage of step length in anterior-posterior (A-P) direction and step width in medial-lateral (M-L) direction; Mean \pm SD. *significant difference $(\mathrm{p}<0.05)$ between lighting conditions.

\subsection{Discussion}

We examined the effects of decreased lighting on reactive balance mechanisms when responding to lean-and-release perturbations. Our findings suggest that when 
environmental and perturbation characteristics are familiar in an obstacle-free environment, decreased lighting does not affect overall balance recovery ability. Individuals were able to utilize vestibular and somatosensory information to detect instability and initiate appropriate responses in both lighting conditions. However, individuals did demonstrate a modified stepping response (greater step length and increased angular velocities) and decreased postural stability in darkness, which may be an effect of decreased visual information.

Decreased ambient lighting did not affect the ability to initiate a response (step onset time) or maximum recoverable perturbation magnitude. Individuals reached a similar maximum lean angle in both lighting conditions $\left(29(7)^{\circ}\right)$, which was slightly higher than maximum lean angle previously reported for young adults $\left(26(5)^{\circ}\right)$ [88]. Our findings suggest that individuals can produce a rapid and successful response without vision by relying on other sensory inputs and prior information to detect and characterize the perturbation. Reactive balance mechanisms require rapid recognition of perturbations to plan and initiate appropriate responses that prevent falling [24]. The somatosensory and vestibular systems provide information regarding the position and acceleration of body segments and can detect minor changes in body position when a perturbation occurs. For example, ankle dorsiflexion and forward acceleration of the head can be detected much faster (80-100 ms latency) than the visual system can sense eye position relative to the environment (200 ms latency) [5, 22, 97, 98]. Balance-correcting responses are initiated 100-120 ms after a perturbation, occurring prior to visual indicators of instability [99]. Therefore, it is possible that initial perturbation detection is based 
primarily on somatosensory and vestibular indicators of instability [98, 99], and is not affected when vision is decreased in darkness.

Interestingly, the dark environment did affect reactive stepping characteristics. In darkness, individuals' reactive steps were greater in length and accompanied by increased knee flexion/extension and hip flexion velocities. We found a step length of 983(154) $\mathrm{mm}$ in Dark, which is higher than previously reported step length values in young adults at a similar lean angle in lit conditions (924(130) $\mathrm{mm}$ ) [88]. Peak hip velocity in Dark $\left(455(26)^{\circ} / \mathrm{s}\right)$ was higher than reported values for hip flexion in normal lighting conditions $\left(\sim 400^{\circ} / \mathrm{s}\right)$, although our observed values for knee flexion in both lighting conditions (Light: $524(16)^{\circ} / \mathrm{s}$; Dark: 558(18) $\%$ s) were lower than reported values in normal light $\left(\sim 650^{\circ} / \mathrm{s}\right)$ [100]; this may have been due to the distribution of lean angles in this study. We calculated angular velocities at lean angles of $14-40^{\circ}$, which could result in a different average knee velocity than a sample mean measured only at $25^{\circ}$. While these increases in step length and angular velocities were not in response to an increase in COM velocity. This may have been a result of a protective response causing overcompensation in the stepping strategy to successfully regain stability. This suggests that vision may play an important role in guiding limb trajectories. Our findings align with previous research examining voluntary stepping trajectories during walking in the absence of visual cues [101, 102]. In studies where vision was removed while stepping over an obstacle, individuals varied their foot placement prior to stepping over the obstacle and increased foot clearance, even when the location of the obstacle was known and the response could be pre-planned [101, 102]. Therefore, although overall response ability was not affected, visual information does appear to play an important role in 
guiding limb movement in real-time, resulting in more conservative reactive stepping strategies when vision is removed.

These changes to reactive stepping that we identified were accompanied by a $12 \%$ decrease in A-P postural stability and increased trunk extension velocity in darkness. Trunk extension occurred after tether release until individuals were able to initiate a stepping response and return to a neutral or flexed trunk position. Increased trunk extension velocity may indicate that individuals had less control over the forward momentum of the pelvis after the tether was released, causing the trunk to extend more rapidly. Decreased control over the forward acceleration of the body immediately after tether release may have contributed to lower postural stability when the step was completed. Although postural stability was still relatively high in both lighting conditions and our sample of young individuals were able to regain control of the COM in darkness in a single step, this may not be possible in older or clinical populations who may have decreased stability.

Our findings provide a valuable first step in our understanding of the influence of decreased ambient lighting conditions on reactive stepping ability. We found that even under the most ideal conditions for balance recovery (known perturbation, obstacle-free environment), young adults free of impairments that would affect balance had modified stepping responses and decreased COM control when online visual information was not accessible in dark environments. Our findings suggest that dark environments can affect balance recovery in all populations and environments and should always be considered when trying to reduce slips, trips, and falls. Home, public, and occupational environments 
with dim lighting could lead to more falls, especially when additional tripping hazards and constraints to reactive balance mechanisms are present (e.g., stairs, room clutter, curbs). Current lighting standards are not based on the amount of light required for balance control, which should be considered in future research to determine optimal levels of light in built environments. Providing adequate lighting could produce safer environments for all populations by minimizing tripping hazards and improving balance control.

While this study provides important foundational information regarding the influence of lighting conditions on reactive stepping ability, it is important to note some limitations. Firstly, perturbations we elicited were not novel or unexpected, therefore differing from losses of balance experienced in real-world settings. Most losses of balance outside the laboratory setting do not allow for any preparation or pre-planning of the response. Participants in this study were familiar with how to respond to the perturbation due to multiple practice trials, which may have affected how they responded when visual information was decreased. Future research regarding reactive balance in dark environments should include perturbations that vary in timing, direction, and magnitude in order to make the response less predictable. Additionally, we elicited perturbations in a clear and unrestricted environment. Environments with obstacles, uneven surfaces, stairs, etc., present more tripping hazards and limit the locations of limb placement during balance recovery (e.g., grasping a handrail, stepping onto a stair). Future research is needed to understand how decreased visual information affects balance recovery when environmental constraints are present and limit the use of a single step response to regain balance. It should also be considered that our sample was young and 
did not have any neurological/physical impairments that affected balance. Dark environments may have larger ramifications for balance in populations with impaired sensory function or reactive balance ability (e.g., older adults, individuals with peripheral neuropathy). Lighting conditions should be considered as a factor during balance recovery in future research, especially when combined with other factors that affect reactive balance (e.g., dual tasking, co-morbidities) [27].

In summary, our findings suggest that decreased ambient lighting does not affect the success of reactive balance responses in healthy, young adults when in a familiar, obstacle-free environment. However, darkness can modify stepping trajectories due to a lack of online visual information and decrease COM control, and could affect balance recovery success when environmental challenges are present (e.g., obstacles, stairs, curbs) or individuals have balance impairments. This study shows the importance of online visual information to guide reactive limb movements, even when pre-planning of the response is possible. Our results provide valuable foundational insights into the use of sensory information during reactive balance control when visual cues are compromised due to decreased lighting. This information can help guide future research on understanding postural control and underlying mechanisms of increased falls in areas of decreased ambient lighting, with the ultimate goal of reducing falls. 


\section{Paper 2 - The effect of decreased ambient lighting on neuromuscular responses while anticipating and responding to forward losses of balance}

\subsection{Introduction}

Falls are responsible for $\sim 10$ million annual injuries in the United States [2]. The balance control system is able to prevent falls by anticipating and reacting to instances of instability [33]. One risk factor for falling is dim lighting [8]. Sensory cues from the visual, vestibular, and somatosensory systems rapidly alert the central nervous system (CNS) when instability is occurring and help plan an appropriate postural response to prevent falling [5]. However, sensory information is not always available due to environmental constraints [35]. Dark environments decrease the availability of visual information, and falls are often reported in areas of dim lighting [11, 12]. Although dim lighting is a risk factor for falling [8], limited research has examined how dark environments mechanistically affect balance control and the ability to prevent a fall when a loss of balance occurs.

Reactive balance mechanisms (or balance recovery) involve the rapid implementation of a motor response that attempts to regain control of balance following a destabilizing perturbation (e.g., a slip or trip) [24]. These responses utilize the trunk and limbs to either generate torques that stabilize the center of mass (COM) within the base of support (BOS) (e.g., rapid arm movements, contracting leg and hip musculature) or change the size/shape of the BOS to accommodate the movement of the COM (e.g., grabbing a handrail, taking a step away from the supporting leg) [27, 33].

The material contained in Chapter 5 is in preparation for submission to Applied Ergonomics. 
Following perturbation detection, the CNS must quickly integrate sensory information regarding perturbation characteristics (e.g., size, direction) and implement a corrective motor response before the COM has moved outside of a recoverable range (latencies as short as 50-110 ms) [103, 104]. Reactive balance mechanisms do not allow time for anticipatory postural adjustments before implementation $[86,105]$, requiring very rapid and complex muscular coordination. The timing and amplitude of neuromuscular responses must also be adapted to meet environmental and task-related constraints [106].

When information from one sensory system is not available, the CNS can rely more on sensory inputs from the available systems to maintain balance [17]. However, previous research indicates that decreased visual information can affect neuromuscular responses during reactive balance $[59,107]$. When visual cues are removed (from wearing a blindfold or closing the eyes), individuals respond to small, translational and tilting platform perturbations with decreased lower limb muscle activation amplitudes, greater co-activation of the shank muscles, and slower muscular adaptation over repeated trials $[59,107]$. However, these studies examined the effect of decreased visual information when a change in the BOS was not required to recover balance (i.e., fixedsupport strategies). Real-world losses of balance often require a larger response involving stepping or grasping to increase the BOS, thereby accommodating the COM (i.e., changein-support strategies) [33].

Visual information may be more important during change-in-support balance strategies compared to fixed-support strategies due to the role of online visual 
information when guiding limb trajectories. When online visual information is removed during gait $[101,102]$ and balance recovery from large perturbations (refer to Paper 1), step location and trajectory are modified even when the response can be pre-planned, resulting in a protective and overcompensated response. Although these findings provide insight into how body movements are altered without visual information, it is unknown how decreased online visual input will affect neuromuscular response characteristics and coordination patterns during reactive stepping.

Existing research suggests that postural responses in the dark may be different than when the eyes are closed due to delayed sensory reweighting when the eyes are open but no visual information is received [78]. During daily life, losses of balance are more likely to occur in a dark environment than a situation where the eyes are closed or covered. Therefore, it is important to understand how reactive balance mechanisms to large perturbations are modified in these situations. Identifying the effects of darkness on the timing, coordination, and activation amplitude of postural muscles can provide insight into why people fall in dark environments, and contribute to the safe design, assessment, and modification of built environments to reduce fall risk.

Our research examined how dark environments affect lower limb muscle activations in young adults performing reactive stepping responses to lean-and-release perturbations. Specifically, we examined the effects of the complete absence of ambient lighting on the timing (muscle onset latencies), amplitude (mean and maximum activations), and coordination (co-contraction indices (CCI), cross-correlation coefficients) of lower limb muscular responses in anticipation and response to these 
perturbations. We hypothesized that individuals would not show any delay in postural responses (muscle onset latencies) due to the long latency of the visual system (200 ms) $[97,104]$. We also hypothesized that individuals would show overcompensated muscular responses in darkness as indicated by increased stiffening/co-contraction of lower limb muscles prior to the perturbation $(\mathrm{CCI})$ and greater, less coordinated muscle activation amplitudes during reactive stepping.

\subsection{Methods}

5.2.1 Participants: This paper was a secondary analysis of the same participants included in Paper 1 (refer to section 4.2.1), with the exception of one participant. One participant was excluded due to an error synchronizing the surface electromyography and motion capture systems. In total, 19 young adults (18-30 y) without muscular or neurological impairments that would affect balance were included to analyze Paper 2 outcomes (mean (SD); age: 23.4(4.5) y; 9 female, 10 male; mass: 75.4(17.4) kg; height: $1.8(0.1) \mathrm{m})$.

5.2.2 Study Protocol: The study protocol is detailed in Paper 1; please refer to section 4.2.2.

5.2.3 Data Acquisition: Bilateral muscle activations were recorded from the tibialis anterior (TA), medial gastrocnemius (MG), rectus femoris (RF), and biceps femoris (BF) using a Delsys or Noraxon 8-channel wireless surface electromyography (EMG) system (Delsys Inc., USA; Noraxon Inc., USA). Due to equipment malfunction of the Delsys system (sampling frequency: $2000 \mathrm{~Hz}$ ), EMG data were collected from 6 
participants using the Noraxon system (sampling frequency: $1500 \mathrm{~Hz}$ ). All statistical comparisons of EMG data were made within participants; each participant used the same system for all of their trials. Electrode placement sites [108] were prepared with skin preparation gel and cleaned with isopropyl alcohol prior to electrode adhesion.

Full-body kinematics were captured using an 11 camera Qualisys 3D motion capture system (Qualisys Inc., Sweden) (sampling frequency: $100 \mathrm{~Hz}$ ). A description of kinematic data acquisition can be found in Paper 1 (Section 4.2.3). EMG data were synced into the Qualisys software using a 5V pulse.

5.2.4 Data Analysis: For the purpose of this study, kinematic data were only used to determine tether release time, toe-off time, and heel-strike time. For a description of how these events were determined, please refer to Paper 1 (Section 4.2.4).

EMG data were demeaned and filtered with a fourth order, zero-lag, bandpass Butterworth filter between $20-450 \mathrm{~Hz}$, full-wave rectified and filtered again with a fourth order, zero-lag, low pass Butterworth filter (cutoff frequency: $10 \mathrm{~Hz}$ ) [109]. EMG analyses were performed in MATLAB (The Mathworks Inc., USA) using custom scripts. All EMG outcomes were calculated at each participant's maximum lean angle where balance could successfully be recovered by taking one step in both Light and Dark (Appendix C). Due to the design of the ramp-up protocol, participants completed only 1 trial in each condition.

Muscle Onset Latencies. Muscle onset latencies after tether release were calculated for all recorded muscles using an algorithm to determine when all activation points of the 
normalized signal within a $25 \mathrm{~ms}$ time window exceeded three standard deviations of the baseline activation within $50 \mathrm{~ms}$ before tether release [110]. Onset times were confirmed through visual inspection [86].

Muscle Activations. The overall maximum activation of each muscle was determined as the peak RMS reached during the 3 failure trials at which participants could not recover balance with 1 step. Muscle activations during all trials were normalized to these peak values (\%MAX). Mean and maximum activations were calculated for 2 time periods: 1) Pre-Perturbation: $0.5 \mathrm{~s}$ before tether release to tether release, 2) Post-Perturbation: tether release to heel-strike. Muscle activations were calculated for both the stepping leg and the standing leg.

Co-Contraction Index (CCI). Lower limb anticipation of the perturbation during the $0.5 \mathrm{~s}$ prior to tether release was evaluated using the co-contraction index $(\mathrm{CCI})$ to quantify the level of co-activation between muscles on the same segment (shank: TAMG; thigh: RF-BF) and muscles on adjacent segments on the same side of the leg (anterior: TA-RF; posterior: MG-BF) [109]. CCI quantifies the level of co-activation between two muscles within a given time period [111], as a composite measure of similarities in the magnitude and timing of the activations $[109,112]$. For each data point, the high $\left(\mathrm{EMG}_{\text {high }}\right)$ and low $\left(\mathrm{EMG}_{\text {low }}\right)$ EMG values were identified based on relative activations of the muscles being compared. CCI was then calculated at each time point as the ratio between muscle activation levels, weighted by the total muscle activity; these values were then summed over the $0.5 \mathrm{~s}$ time period using Equation 5.1, where ' $N$ ' is the 
total number of data points (equal to the sampling frequency), and ' $i$ ' indicates each data point [113].

$$
\text { Equation 5.1: CCI }=\sum_{i=1}^{N} \frac{\mathrm{EMG}_{\mathrm{low}_{i}}}{\mathrm{EMGG}_{\mathrm{high}_{i}}}\left(\mathrm{EMG}_{\mathrm{low}_{i}}+\mathrm{EMG}_{\mathrm{high}_{i}}\right)
$$

Higher CCI values indicate more co-contraction of muscles and increased 'stiffening' of segments $[114,115]$ in anticipation of the perturbation.

Cross-correlation. Cross-correlation was used to analyze the effects of lighting condition on muscular coordination during the stepping response (tether release to heelstrike). Cross-correlation quantifies the relationship between 2 time-varying EMG signals $(x(\mathrm{t})$ and $y(\mathrm{t}))$, where a positive cross-correlation coefficient $\left(\mathrm{R}_{x y}\right)$ indicates both muscles are contracting at the same time and a negative coefficient indicates that one muscle contracts as the other relaxes [116]. We analyzed the coordination between muscles on the same segment (shank: TA-MG; thigh: RF-BF) and muscles on the same side of adjacent segments (anterior: TA-RF; posterior: MG-BF). Cross-correlation produces a function $\mathrm{R}_{x y}[\tau]$ which indicates muscular coordination at incremental time points $(\tau)$ relative to the sampling frequency $(f s)$ during the response (Equation 5.2) [116]. We calculated the cross-correlation coefficient at a time lag $(\tau)$ of zero to determine the coordination between two muscles as they activated in real-time.

$$
\text { Equation 5.2: } \mathrm{R}_{x y}[\tau]=\frac{\frac{1}{N} \sum_{i=1}^{N}\left(x_{i}-\bar{x}\right)\left(y_{i+\tau f_{s}}-\bar{y}\right)}{\frac{1}{N} \sqrt{\sum_{i=1}^{N}\left(x_{i}-\bar{x}\right)^{2} \sum_{i=1}^{N}\left(y_{i}-\bar{y}\right)^{2}}}
$$


Statistical Analysis. Paired sample t-tests were used to analyze differences in mean and maximum muscle activations between lighting conditions (Light, Dark) in 2 time periods (Pre-Perturbation: $0.5 \mathrm{~s}$ before tether release to tether release; Post-Perturbation: tether release to heel-strike). The effects of lighting condition on muscle onset latencies, $\mathrm{CCI}$, and cross-correlation coefficients were also analyzed using paired sample t-tests. The differences in dependent variables between lighting conditions were assessed for normality using Shapiro-Wilk tests to meet the assumptions for paired sample t-tests. Hotilly's $\mathrm{T}^{2}$ test was also used to analyze the effect of lighting on all muscles (muscle onset latencies, mean and maximum activations) and muscle pairings (CCI, crosscorrelation coefficients) at once. Pearson correlations were used to analyze the relationship between variables that were significantly affected by lighting by assessing the changes in each variable between Light and Dark, including correlations with variables included in Paper 1 that were significantly affected by lighting. Statistical analyses were performed using SPSS v. 26 (IBM Corp, SPSS Statistics, USA). Alpha was set to 0.05 for all tests.

\subsection{Results}

5.3.1 Muscle Onset Latencies: Many participants activated lower limb muscles prior to tether release, likely due to anticipation of the perturbation. Muscle onset thresholds occurred before tether release for 3\% of trials in the stepping leg TA, $8 \%$ of trials in the standing leg TA, $26 \%$ of trials in the stepping leg MG, $24 \%$ of trials in the standing leg MG, $0 \%$ of trials in the stepping leg RF, $3 \%$ of trials in the standing leg RF, $11 \%$ of trials in the stepping leg BF, and $5 \%$ of trials in the standing leg BF. Trials with 
negative onset latencies were excluded from statistical testing resulting in the following sample sizes for each muscle (Stepping TA: $n=18$; Standing TA: $n=16$; Stepping MG: n=12; Standing MG: $n=11$; Stepping RF: $n=19$; Standing RF: $n=18$; Stepping BF: $n=16$; Standing BF: $\mathrm{n}=17)$.

Multivariate $\left(\mathrm{T}^{2}(8,14)=1.61, \mathrm{p}=0.95\right)$ and individual $\mathrm{t}$-tests $(\mathrm{p}>0.05)$ did not indicate any effects of lighting on muscle onset latencies (Figure 5.1). Onset latencies were shortest in the BF (Step: 32(37) ms; Stand: 38(32) ms), followed by the MG (Step: 38(40) ms; Stand: 38(34) ms), TA (Step: 38(40) ms; Stand: 47(37) ms), and RF (Step: 88(30) ms; Stand: 81(34) ms). Paired sample t-test results and effect size for all variables can be found in Appendix E. 

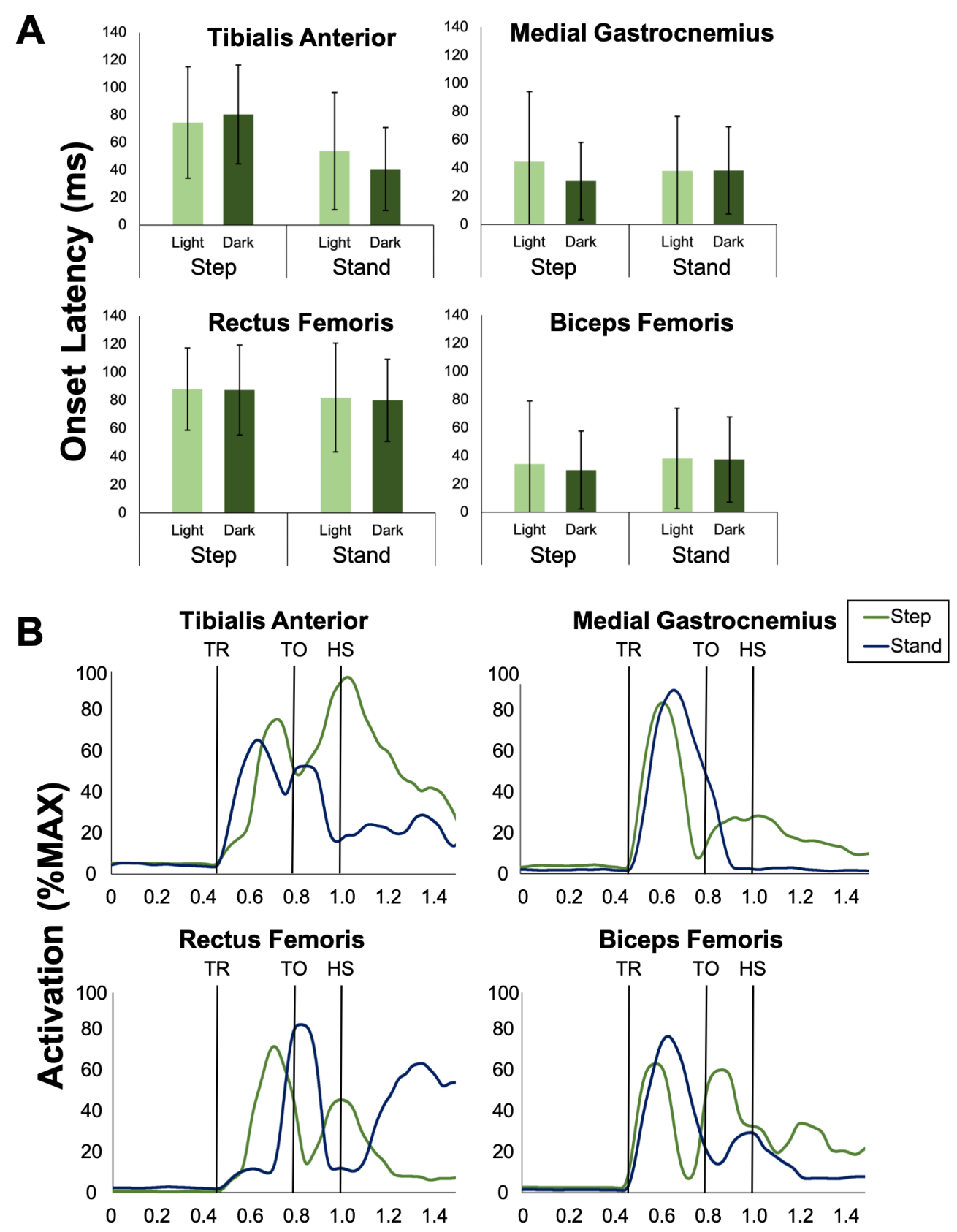

Time (s)

Figure 5.1 (A) Muscle onset latency times (ms) for tibialis anterior (TA), medial gastrocnemius (MG), rectus femoris (RF), and biceps femoris (BF); Mean \pm SD. (B) Filtered and normalized muscle activations (\%MAX) during reactive stepping in single participant (Light condition). TR: tether release; TO: toe-off; HS: heel-strike. 
5.3.2 Muscle Activations: Multivariate testing including all muscles indicated that mean $\left(\mathrm{T}^{2}(16,20)=7.23, \mathrm{p}=0.87\right)$ and maximum $\left(\mathrm{T}^{2}(16,20)=6.752, \mathrm{p}=0.30\right)$ muscle activations were not different between lighting conditions. Paired sample t-tests comparing the activation of individual muscles indicated that mean activation of the TA on the standing leg was significantly larger in Dark (41(11)\%MAX) than Light (36(11)\%MAX) post-perturbation ( $\mathrm{p}=0.04)$ (Figure 5.2). There was a significant correlation between this change in TA activation and changes in hip flexion velocity $(\mathrm{r}(17)=0.52, \mathrm{p}=0.02)$ and trunk extension velocity $(\mathrm{r}(16)=-0.49, \mathrm{p}=0.04)$ between lighting conditions. Lighting did not have a significant effect on mean or maximum activation of any other muscles pre- or post-perturbation ( $\mathrm{p}>0.05$ ) (Figures 5.2 and 5.3). Both pre- and post-perturbation, the highest activations occurred in the MG (Pre-Perturbation: 44(22)\%MAX; Post-Perturbation: 80(17)\%MAX), followed by the BF (Pre: 31(19)\%MAX; Post: 69(25)\%MAX), RF (Pre: 15(7)\%MAX; Post: 63(27)\%MAX), and TA (Pre: 15(14)\%MAX; Post: 62(16)\%MAX). 


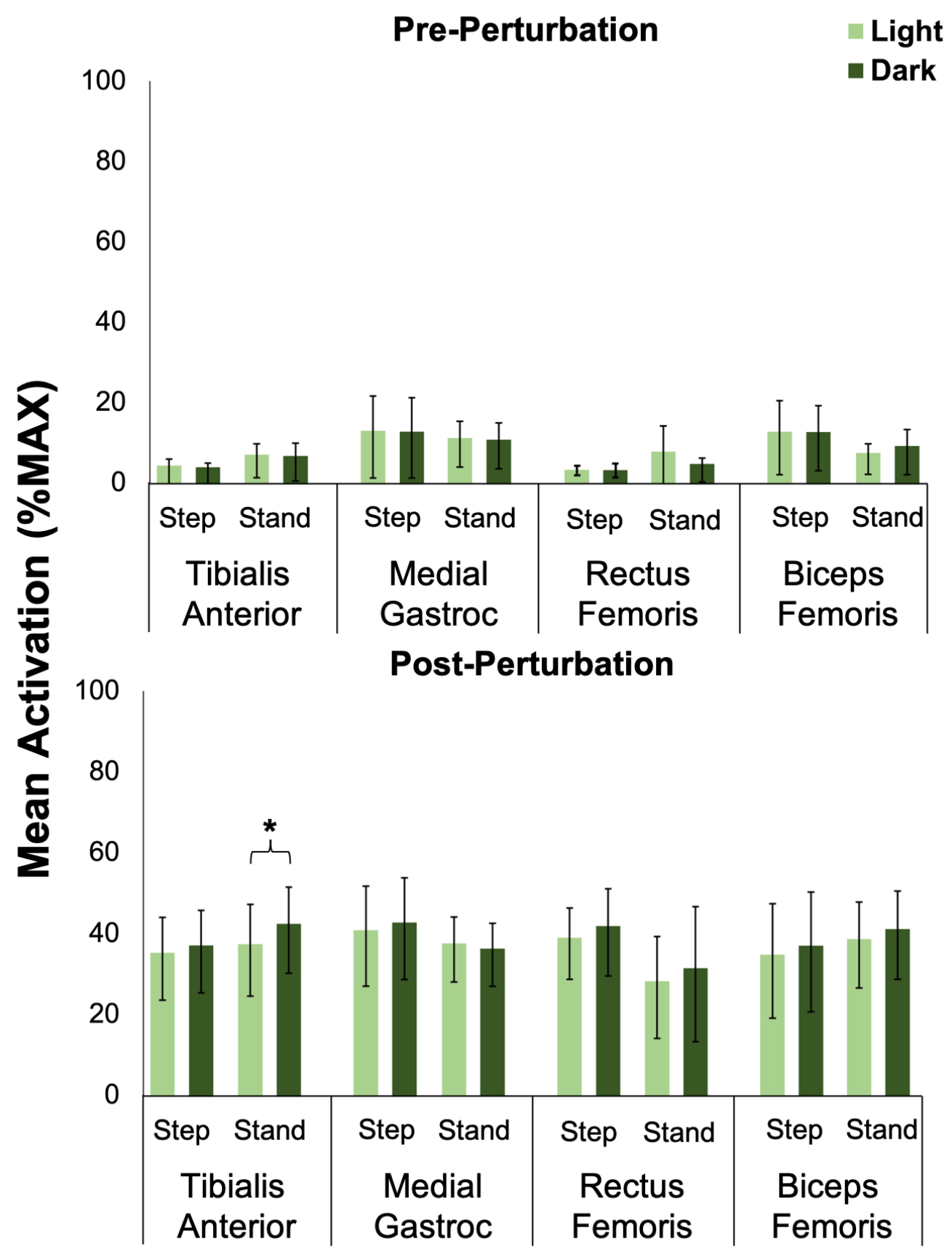

Figure 5.2 Mean activation of the tibialis anterior (TA), medial gastrocnemius (MG), rectus femoris (RF), and biceps femoris (BF) on the stepping and standing leg preperturbation ( $0.5 \mathrm{~s}$ before tether release to tether release) and post-perturbation (tether release to heel-strike); Mean \pm SD. *significant effect of lighting $(\mathrm{p}<0.05)$. 


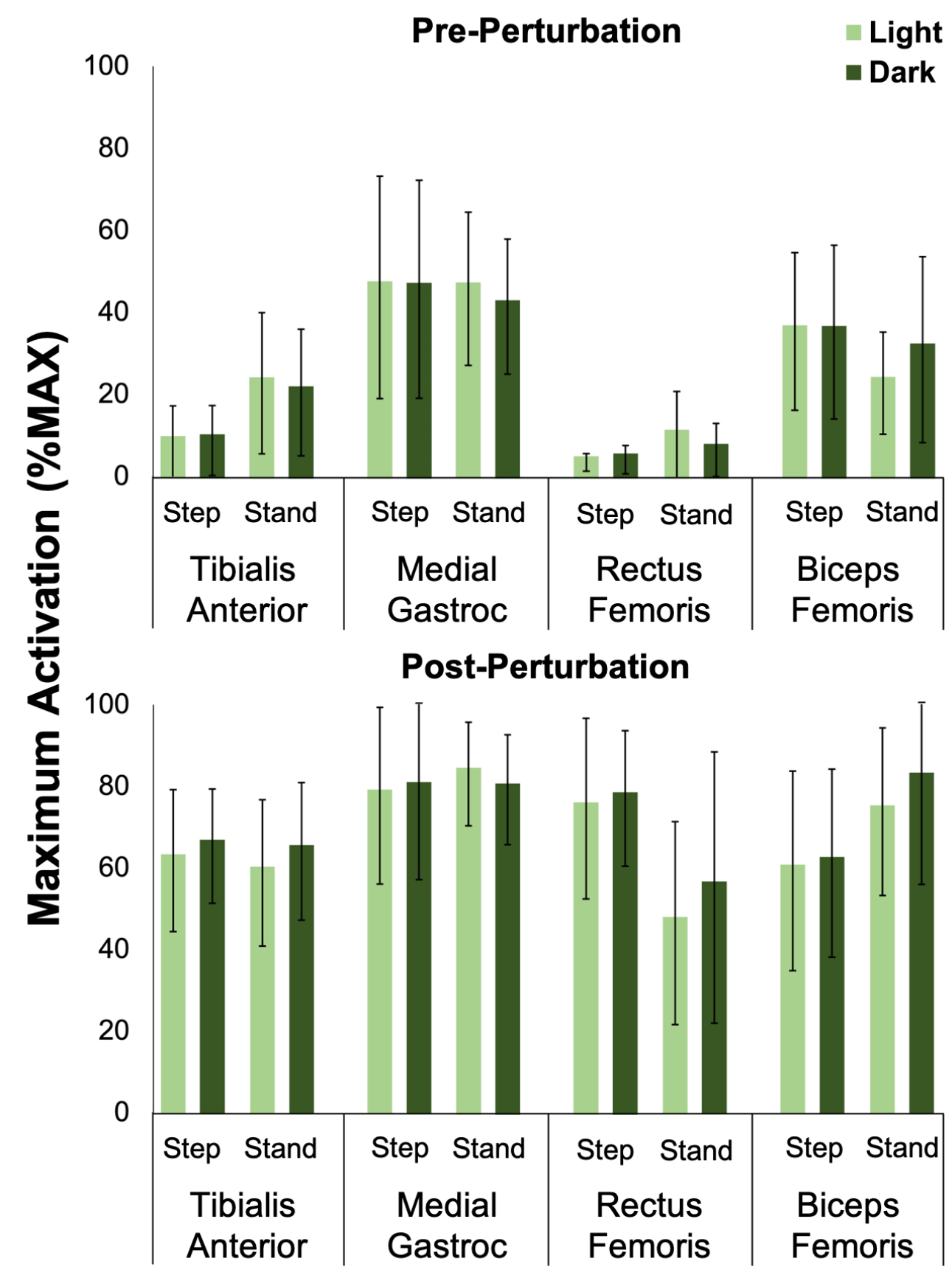

Figure 5.3 Maximum activation of the tibialis anterior (TA), medial gastrocnemius $(\mathrm{MG})$, rectus femoris (RF), and biceps femoris (BF) on the stepping and standing leg preperturbation $(0.5 \mathrm{~s}$ before tether release to tether release) and post-perturbation (tether release to heel-strike); Mean $\pm \mathrm{SD}$. * significant effect of lighting $(\mathrm{p}<0.05)$. 
5.3.3 Co-Contraction Index: Multivariate testing indicated no effect of lighting on all muscle pairings $\left(\mathrm{T}^{2}(8,26)=3.38, \mathrm{p}=0.66\right)$. Individual t-tests also indicated that lighting did not affect $\mathrm{CCI}$ in the $0.5 \mathrm{~s}$ prior to tether release in the shank (Step: 263(246)\%MAX; Stand: 121(109)\%MAX), thigh (Step: 524(718)\%MAX; Stand: 163(217)\%MAX), anterior leg muscles (Step: 38(55)\%MAX; Stand: 85(147)\%MAX), or posterior leg muscles (Step: 274(290)\%MAX; Stand: 177(218)\%MAX) (p>0.05) (Figure 5.4).

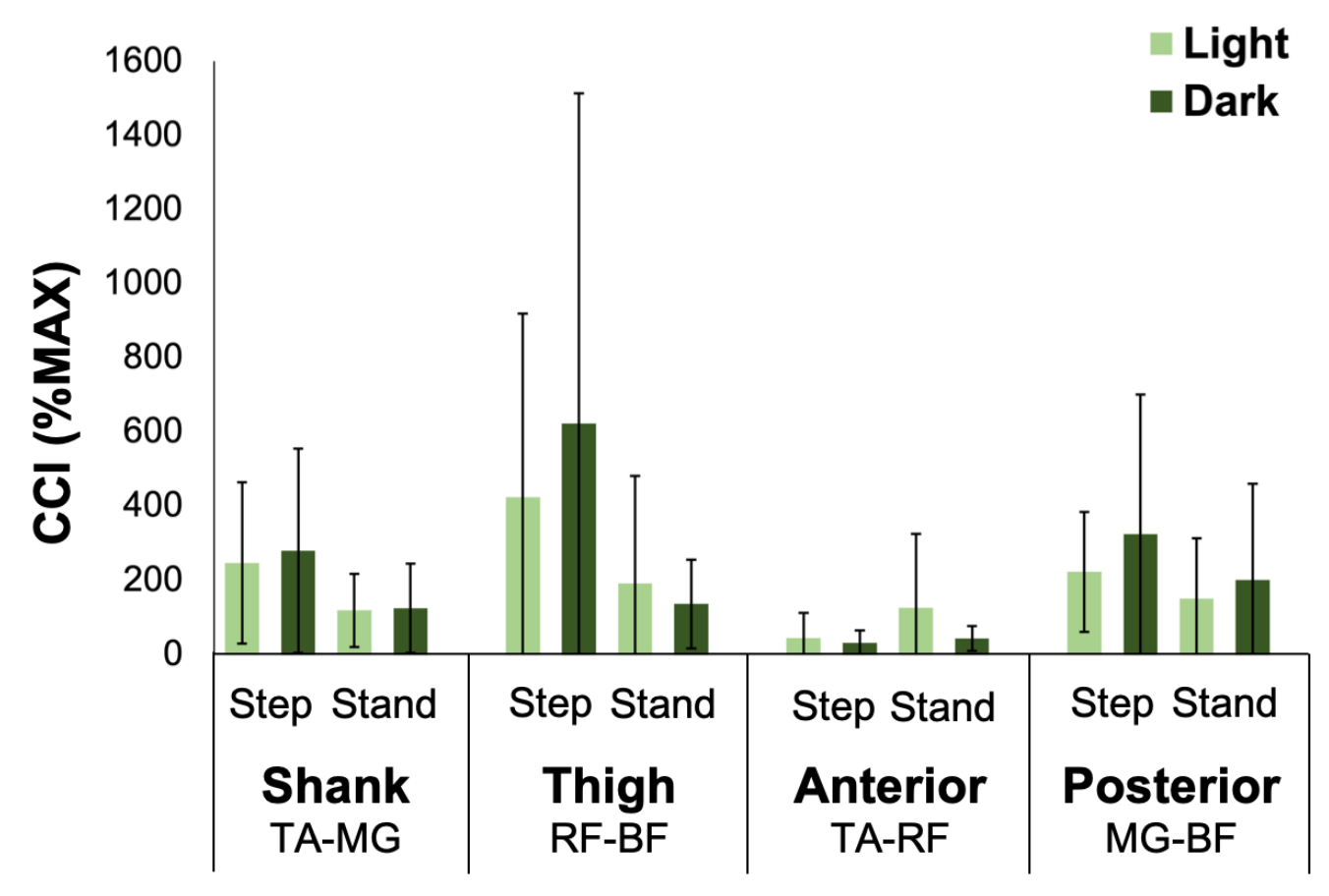

Figure 5.4 Co-Contraction Index (CCI) in the shank segment (TA-MG), thigh segment (RF-BF), anterior muscles (TA-RF), and posterior muscles (MG-BF); Mean $\pm \mathrm{SD}$.

5.3.4 Cross-Correlation: Multivariate testing including all muscle pairings indicated no effect of lighting on zero-lag cross-correlation coefficients $\left(\mathrm{T}^{2}(8,28)=2.90\right.$, $\mathrm{p}=0.74)$. Individual $\mathrm{t}$-tests indicated that cross-correlation coefficients were significantly affected by lighting in the thigh segment (RF-BF) on the standing leg $(\mathrm{p}=0.02)$. Cross- 
correlation coefficients were significantly higher in Light $(0.17(0.32))$ than Dark $(0.01(0.29))$. Further examination of the data revealed that a mean correlation coefficient close to zero in darkness resulted from 8 participants having positive correlation coefficients between the RF and BF, and 11 participants having negative coefficients, with a mean close to zero (Figure 5.5). Overall, most cross-correlation coefficients were positive, indicating that participants were activating both paired muscles at the same time during the stepping response.

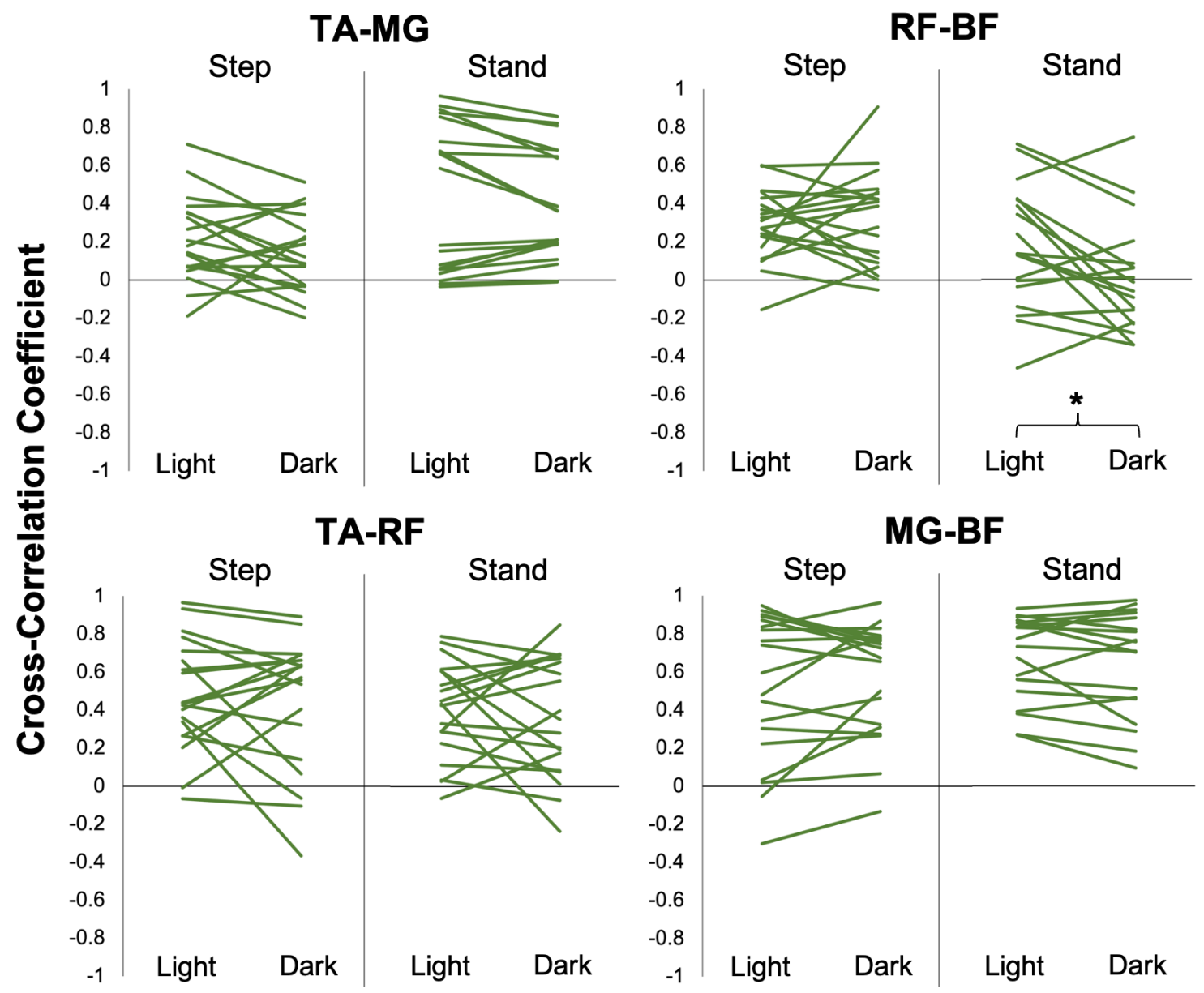

Figure 5.5 Variability in zero-lag cross-correlation coefficients between shank (TA-MG), thigh (RF-BF), anterior (TA-RF), and posterior (MG-BF) muscles on stepping and standing leg. Each line indicates individual participant. *significant effect of lighting $(\mathrm{p}<0.05)$. 


\subsection{Discussion}

We examined the effects of decreased ambient lighting on lower limb neuromuscular responses to forward lean-and-release perturbations. During the dark condition, participants demonstrated increased TA activation accompanied by increased coordination of the thigh muscles. However, the absence of ambient lighting did not affect muscle onset latencies, co-contraction prior to perturbation onset, or activation amplitudes of the MG, RF, or BF. These findings suggest that although decreased visual information may affect some muscle activation characteristics during reactive balance, it does not affect the ability to initiate a reactive stepping mechanism or the overall goal of recovering balance.

Absence of ambient lighting did affect muscle activation during reactive stepping execution. Although lighting condition did not affect muscle activations prior to the perturbation (mean and maximum activation, co-contraction), some muscle activations later in the stepping response were different in darkness. Following the perturbation, individuals showed increased mean activation of the standing leg TA (5\% increase in darkness). Previous studies examining neuromuscular responses with the eyes closed have also found that individuals show increased shank muscle activation when responding to platform translations with feet-in-place strategies [59, 117]. Sozzi et al. reported a 3\% increase in normalized mean TA activation with the eyes closed following habituation to small support surface translations [59], and a 5\% increase during perturbed tandem stance [117]. However, these values were much smaller (4 vs. 1\%MAX; 9 vs. 4\%MAX, respectively) compared to the values we obtained during stepping (41 vs $36 \% \mathrm{MAX})$. These results suggest that individuals may overcompensate the muscular 
response necessary to recover balance without visual information, particularly by increasing shank muscle activation on the legs that are in contact with the ground (i.e., part of the BOS). Individuals in our study also showed statistically lower crosscorrelation coefficients between the RF and BF in darkness. A lower coefficient in the thigh segment potentially indicates a more coordinated activation pattern between the RF and BF rather than an 'all-on' strategy. However, given the variability in these data, these changes will require further investigation to confirm their occurrence.

Muscle onset latencies following the perturbation were not affected in dark environments. Similar findings have been reported during postural perturbations when the eyes are closed [59]. This suggests that perturbation detection is not delayed when visual information is unavailable. Although vision is known to play an important role in postural control [45], the CNS potentially relies more on the somatosensory and vestibular systems to detect perturbations because they are faster conducting systems [22, 97]. Previous research suggests that postural responses may be initiated based on somatosensory and vestibular cues, particularly the stretching of muscle spindles in the feet and ankles [118-120]. While the visual system has a latency of $\sim 200 \mathrm{~ms}$, we found muscle onset latencies to be $\sim 30-100 \mathrm{~ms}$, indicating that a neuromuscular response was already implemented before the visual system would have been able to detect the perturbation. Although we found relatively short onset latencies, most reported values for reactive onset latencies in postural muscles are less than $200 \mathrm{~ms}[104,120,121]$, indicating that postural responses are initiated prior to visual indicators of instability. 
This study was mainly limited by the predictability of the perturbation. Participants knew they would experience a perturbation after leaning forward and could practice their response during practice trials, leading to short or negative muscle onset latencies [103]. Additionally, the release of the tether via switch produced a clicking sound that may have provided a cue for participants that they would be released. Real-world slips and trips are often unexpected and novel, not allowing for any anticipation or preparation. Participants in this study may have shown adapted muscular responses that do not accurately represent muscle activation amplitudes that occur during initial exposure to an unexpected perturbation. Future research should utilize methods that make perturbations more variable and unpredictable in dark environments, such as providing mental distractions to the participant or including unexpected catch trials without a perturbation.

Although the differences in neuromuscular responses between lighting conditions were small, we still found that dark environments can affect reactive balance. It is notable that young adults free of balance impairments can still be affected by conditions of decreased ambient lighting during a relatively simple reactive strategy (e.g., anticipated perturbation, obstacle-free environment). Losses of balance can be more challenging to respond to in real-world environments that contain slippery/uneven surfaces, stairs, handrails, room clutter, etc. and require specific limb trajectories to recover balance (e.g., stepping onto a stair). Additionally, environmental layout is not always familiar, such as entering an environment that is already dark. Differences in neuromuscular responses could be more pronounced during balance recovery in real-world dark environments when perturbations are unexpected and require a more complex response, especially in older adults or clinical populations that have sensory/balance impairments. Reduced 
ambient lighting affects balance recovery even in the most ideal conditions, and could affect the ability to prevent a fall when reactive balance becomes more challenging.

In conclusion, the findings of this study suggest that dark environments can affect muscle activations during reactive stepping responses to anticipated perturbations in young adults without affecting perturbation detection. Decreased access to visual information could have more pronounced effects when obstacles/reactive stepping barriers are present and losses of balance are novel. Further research in this area is necessary to understand how dark environments affect the ability to control balance in a variety of environments and populations in order to design future environments with lighting that minimizes fall risk. 


\section{Contributions and Future Directions 6.1 Summary of Outcomes}

The purpose of this thesis was to examine the effects of decreased ambient lighting on reactive balance mechanisms that require a stepping response. I evaluated the effects of lighting condition on reactive stepping parameters, $\mathrm{COM}$ control, and neuromuscular response characteristics. This research is novel because to my knowledge, it was the first study that has examined reactive stepping in dark environments. Although previous research has provided valuable information about the effects of visual cue removal on postural control and gait by closing or covering the eyes $[9,10,15,46,53,57]$, there is limited knowledge about the effects of dark environments on reactive balance.

My findings suggest that environments with decreased ambient lighting do not have a robust overall effect on reactive stepping mechanisms, but can result in some modifications to reactive stepping and decreased COM control. Specifically, I found that during reactive stepping in the dark ambient lighting condition, individuals responded with greater step length, knee/hip/trunk angular velocities, standing leg TA activation, and thigh coordination. These modifications were accompanied by decreased A-P stability at heel-strike. Individuals showed no differences in the ability to initiate a response or successfully recover from perturbations.

\subsection{Real-World Applications}

These findings are notable because I found differences between lighting conditions during a simple reactive balance task in a young sample free of balance impairments. Perturbation and sample characteristics provided the most ideal conditions for balance 
recovery. Reactive stepping could be anticipated, pre-planned, and completed without having to consider any environmental constraints (e.g., stairs, room-clutter, uneven surfaces), and individuals did not have any neurological/physical impairments that affected their ability to detect or respond to the perturbation. Lighting conditions could have more pronounced effects on reactive balance mechanisms when two major cases occur in real-world scenarios: 1) When losses of balance require complex responses due to environmental characteristics, and 2) When older individuals or clinical populations have sensory/physical impairments that affect reactive balance or reliance on visual information.

6.2.1 Environmental Characteristics: The testing environment in this study was level and obstacle-free and the stepping foot could be placed essentially anywhere (assuming that the location of the step would be appropriate to accommodate the COM). This environment allowed us to examine the detection of instability under ideal conditions without any confounding factors. However, this is not always the case in real environments which may contain stairs, curbs, slippery surfaces, or obstacles on the ground. These current findings and previous research suggest online visual information is important to guide the stepping foot during the swing and landing phase [101, 102]. In an environment with obstacles/stairs that limit the trajectory and placement of reactive steps, modifications caused by a lack of visual information could lead to contact with obstacles or inappropriate step placements that limit successful balance recovery. Additionally, maneuvering limbs around environmental constraints could be more challenging when 
the layout of the environment is unknown, such as entering an environment which is already dark.

6.2.2 Older and Clinical Populations: The participants in this study were young and had no neurological/physical impairments that affected their balance. Lighting still affected young adults, and may have more pronounced effects on individuals with balance or sensory impairments. Older adults and clinical populations generally show worse performance on reactive balance tasks than young or unaffected adults $[88,122-$ 124]. Older adults and individuals post-stroke are more likely than young adults to take a multi-step response to a given perturbation [123, 125-128], often stepping laterally towards the unsupported side following an initial step [123]. Maki and McIlroy (2006) suggest that falls in older adults may occur more often due to problems controlling the swing/landing phase rather than step initiation [123, 124, 129]. As discussed in section 6.2.1, step trajectory and placement location can be modified in dark environments and limited by environmental constraints. Individuals who already have trouble taking controlled and stable reactive steps in well-lit environments could be challenged even more in dark environments without visual information as a guide. Additionally, some individuals with sensory impairments from diabetes/peripheral neuropathy or stroke show delayed postural reactions due to impaired perturbation detection [120, 122, 127]. Individuals with impaired somatosensory function or vestibular disorders may rely more on vision for perturbation detection and show differences in response initiation in dark environments. 


\subsection{Implications for Future Research}

I found that lighting can affect reactive balance and could have more dangerous implications in complex environmental conditions or populations with sensory/balance impairments. Dim lighting is a risk factor for falling [8], yet lighting conditions are not included in models of reactive balance [27]. Lighting conditions should be included as an environmental factor that affects reactive balance and future research should consider the effects of lighting when other factors are present, such as handrails, dual-tasking, or peripheral neuropathy (Figure 6.1).

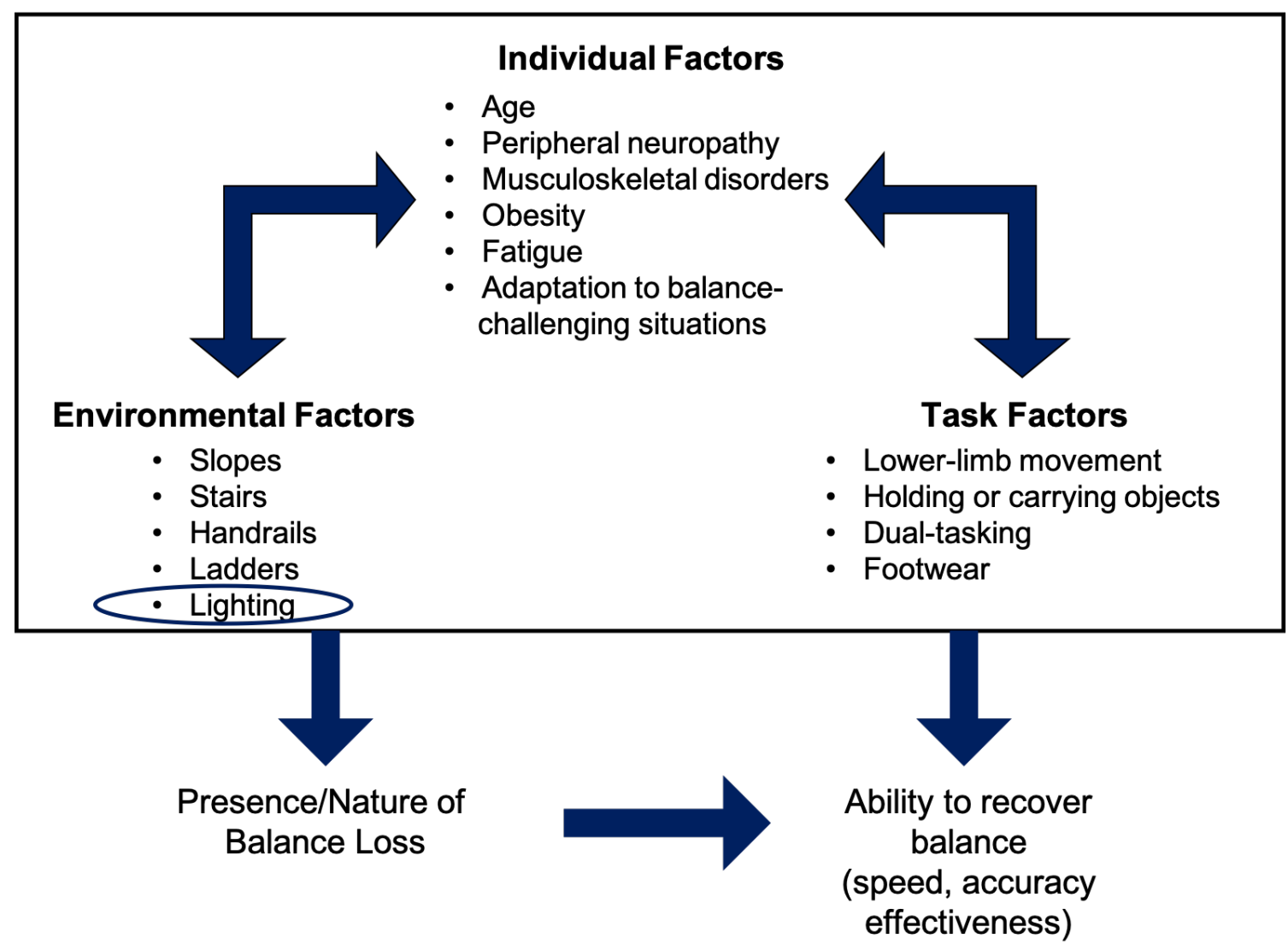

Figure 6.1 Individual, environmental, and task factors that influence balance recovery ability and balance loss (developed from Komisar et al. (2019) [27]). 


\subsection{Implications for Lighting Standards}

This study examined differences in reactive balance between a fully illuminated and completely dark room. I found that complete darkness resulted in modified reactive stepping mechanisms and stability. This fundamental step toward understanding the effects of lighting conditions on reactive balance demonstrates that there is a need to develop evidence-based fall-related lighting standards for built environments and public spaces.

Lighting standards for public buildings and offices in the United States are about 100-300 Lux based on the purpose of the room, however lighting standards in walkways and stairs can be as low as 11-30 Lux $[63,64,130]$. It is unknown if these are standards are appropriate levels of light to avoid the effects of darkness on balance recovery, as no existing research has examined reactive balance in incremental levels of light. Moving forward, this is an important area for future research in order to determine if current building codes recommend lighting levels for optimal balance control and minimal fall risk.

Evidence-based lighting standards are needed because lighting conditions can affect multiple aspects of balance recovery. Table 6.1 categorizes environmental factors that affect balance recovery based on whether they affect the ability to avoid balance loss, the speed of the response, accuracy of limb movements, or effectiveness of balance recovery [27]. I have summarized how decreased lighting conditions can affect reactive balance by adding dim lighting to this table. Environmental tripping hazards increase the risk of balance loss [131] and may be less visible in dim lighting conditions, decreasing 
the ability to avoid balance loss. Additionally, my findings suggest that dim lighting can affect the accuracy of lower limb movements during balance recovery. Although I examined modifications in lower-limb responses, future research could examine if similar effects apply to the accuracy of arm movements (e.g., reaching for a handrail). Lastly, I suggest that dim lighting could affect the effectiveness of balance recovery. Although individuals in this study could still produce effective balance recovery responses in the dark condition, they had decreased A-P stability indicating a change in COM control. Decreased COM control could increase the risk of falling, particularly in individuals with lower stability or balance impairments. Modifications to the swing/landing phase of stepping could also decrease balance recovery effectiveness in cases where obstacles need to be avoided.

Table 6.1. Proposed addition of dim lighting to environmental balance recovery factors (developed from Komisar et al. (2019) [27]).

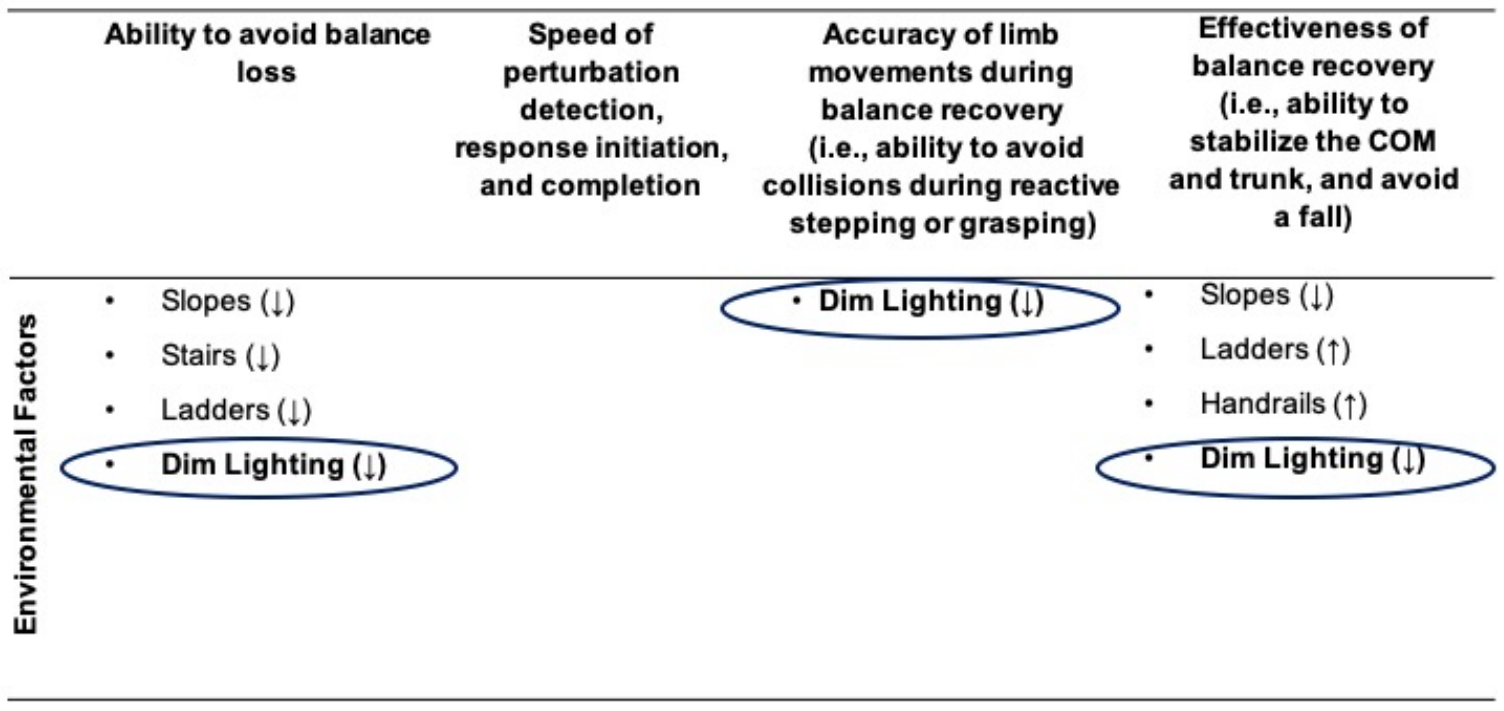

\subsection{Limitations}

This study was mainly limited by the nature of the perturbation. Unlike real-world losses of balance, the perturbations elicited in this study were predictable and repeated, 
allowing for adaptation to the perturbation that might not accurately represent reactive responses to a novel and unexpected loss of balance. It would be beneficial for future research to incorporate methods to make perturbations more variable (e.g., multidirectional, unexpected magnitude) and reduce anticipation in participants (e.g., catch trials, mental distraction). 


\section{Reference List}

1. Pollock, A.S., et al., What is balance? Clinical Rehabilitation, 2000. 14(4): p. 402-406.

2. Verma, S.K., et al., Falls and fall-related injuries among community-dwelling adults in the United States. PLos One, 2016. 11(3): p. e0150939.

3. Stevens, J.A. and E.D. Sogolow, Gender differences for non-fatal unintentional fall related injuries among older adults. Injury Prevention, 2005. 11(2): p. 115119.

4. Galet, C., et al., Fall injuries, associated deaths, and 30-day readmission for subsequent falls are increasing in the elderly US population: a query of the WHO mortality database and National Readmission Database from 2010 to 2014. Clinical Epidemiology, 2018. 10: p. 1627.

5. Winter, D.A., Human balance and posture control during standing and walking. Gait \& Posture, 1995. 3(4): p. 193-214.

6. Shumway-Cook, A. and M.H. Woollacott, Motor control: translating research into clinical practice. 2007: Lippincott Williams \& Wilkins.

7. Horak, F.B., Clinical measurement of postural control in adults. Physical Therapy, 1987. 67(12): p. 1881-1885.

8. Center for Disease Control, Risk Factors for Falls. 2017. Retrieved from https://www.cdc.gov/steadi/pdf/STEADI-FactSheet-RiskFactors-508.pdf

9. Buchanan, J.J. and F.B. Horak, Emergence of postural patterns as a function of vision and translation frequency. Journal of Neurophysiology, 1999. 81(5): p. 2325-2339.

10. Jilk, D.J., S.A. Safavynia, and L.H. Ting, Contribution of vision to postural behaviors during continuous support-surface translations. Experimental Brain Research, 2014. 232(1): p. 169-180.

11. Haslam, R. and T.A. Bentley, Follow-up investigations of slip, trip and fall accidents among postal delivery workers. Safety Science, 1999. 32(1): p. 33-47.

12. Mion, L.C., et al., Falls in the rehabilitation setting: incidence and characteristics. Rehabilitation Nursing, 1989. 14(1): p. 17-22.

13. Horak, F. and J. Macpherson, Handbook of physiology. Exercise: regulation and integration of multiple systems. 1996.

14. Scholz, J., et al., Motor equivalent control of the center of mass in response to support surface perturbations. Experimental Brain Research, 2007. 180(1): p. 163-179.

15. Chien, J.H., et al., Locomotor sensory organization test: a novel paradigm for the assessment of sensory contributions in gait. Annals of Biomedical Engineering, 2014. 42(12): p. 2512-2523.

16. Day, B.L. and J. Cole, Vestibular-evoked postural responses in the absence of somatosensory information. Brain, 2002. 125(9): p. 2081-2088.

17. Peterka, R., Sensorimotor integration in human postural control. Journal of Neurophysiology, 2002. 88(3): p. 1097-1118.

18. Alberts, B.B., et al., Bayesian quantification of sensory reweighting in a familial bilateral vestibular disorder (DFNA9). Journal of Neurophysiology, 2018. 119(3): p. 1209-1221. 
19. Deneve, S. and A. Pouget, Bayesian multisensory integration and cross-modal spatial links. Journal of Physiology-Paris, 2004. 98(1-3): p. 249-258.

20. Kabbaligere, R., B.-C. Lee, and C.S. Layne, Balancing sensory inputs: Sensory reweighting of ankle proprioception and vision during a bipedal posture task. Gait \& Posture, 2017. 52: p. 244-250.

21. Black, F., et al., Abnormal postural control associated with peripheral vestibular disorders. Progress in Brain Research, 1988. 76: p. 263-275.

22. Dietz, V., M. Trippel, and G. Horstmann, Significance of proprioceptive and vestibulo-spinal reflexes in the control of stance and gait. Advances in Psychology, 1991. 78: p. 37-52.

23. Massion, J., Movement, posture and equilibrium: interaction and coordination. Progress in Neurobiology, 1992. 38(1): p. 35-56.

24. Maki, B.E. and W.E. McIlroy, The role of limb movements in maintaining upright stance: the "change-in-support” strategy. Physical Therapy, 1997. 77(5): p. 488507.

25. Maki, B.E. and W.E. McIlroy, Cognitive demands and cortical control of human balance-recovery reactions. 2007. 114(10): p. 1279-1296.

26. Mcllroy, W. and B. Maki, Influence of destabilization on the temporal characteristics of "volitional" stepping. Journal of Motor Behavior, 1996. 28(1): p. 28-34.

27. Komisar, V., W.E. McIlroy, and C.A. Duncan, Individual, task, and environmental influences on balance recovery: A narrative review of the literature and implications for preventing occupational falls. IISE Transactions on Occupational Ergonomics and Human Factors, 2019. 7(2): p. 1-41.

28. Rietdyk, S., et al., Balance recovery from medio-lateral perturbations of the upper body during standing. Journal of Biomechanics, 1999. 32(11): p. 11491158 .

29. McIlroy, W.E. and B.E. Maki, Early activation of arm muscles follows external perturbation of upright stance. Neuroscience Letters, 1995. 184(3): p. 177-180.

30. Cheng, K.B., Y.-C. Huang, and S.-Y. Kuo, Effect of arm swing on single-step balance recovery. Human Movement Science, 2014. 38: p. 173-184.

31. Roos, P.E., et al., The role of arm movement in early trip recovery in younger and older adults. Gait \& Posture, 2008. 27(2): p. 352-356.

32. Cheng, K.B., K.-M. Wang, and S.-Y. Kuo, Role of arm motion in feet-in-place balance recovery. Journal of Biomechanics, 2015. 48(12): p. 3155-3162.

33. Maki, B.E., W.E. Mcilroy, and G.R. Fernie, Change-in-support reactions for balance recovery. IEEE Engineering in Medicine and Biology Magazine, 2003. 22(2): p. 20-26.

34. Sarraf, T.A., D.S. Marigold, and S.N. Robinovitch, Maintaining standing balance by handrail grasping. Gait \& Posture, 2014. 39(1): p. 258-264.

35. Horak, F.B., Postural orientation and equilibrium: what do we need to know about neural control of balance to prevent falls? Age and Ageing, 2006. 35(suppl_2): p. ii7-iil1.

36. Crenshaw, J.R., et al., The discriminant capabilities of stability measures, trunk kinematics, and step kinematics in classifying successful and failed compensatory 
stepping responses by young adults. Journal of Biomechanics, 2012. 45(1): p. 129-133.

37. Mackey, D.C. and S.N. Robinovitch, Postural steadiness during quiet stance does not associate with ability to recover balance in older women. Clinical Biomechanics, 2005. 20(8): p. 776-783.

38. McIlroy, W.E. and B.E. Maki, Age-related changes in compensatory stepping in response to unpredictable perturbations. The Journals of Gerontology Series A: Biological Sciences and Medical Sciences, 1996. 51(6): p. M289-M296.

39. Crenshaw, J.R. and M.D. Grabiner, The influence of age on the thresholds of compensatory stepping and dynamic stability maintenance. Gait \& Posture, 2014. 40(3): p. 363-368.

40. Jensen, J.L., L.A. Brown, and M.H. Woollacott, Compensatory stepping: The biomechanics of a preferred response among older adults. Experimental Aging Research, 2001. 27(4): p. 361-376.

41. Sawers, A., et al., Neuromuscular responses differ between slip-induced falls and recoveries in older adults. Journal of Neurophysiology, 2017. 117(2): p. 509-522.

42. Merrill, Z., A.J. Chambers, and R. Cham, Arm reactions in response to an unexpected slip -Impact of aging. Journal of Biomechanics, 2017. 58: p. 21-26.

43. Bueno-Cavanillas, A., et al., Risk factors in falls among the elderly according to extrinsic and intrinsic precipitating causes. European Journal of Epidemiology, 2000. 16(9): p. 849-859.

44. Gaerlan, M.G., et al., Postural balance in young adults: the role of visual, vestibular and somatosensory systems. Journal of the American Academy of Nurse Practitioners, 2012. 24(6): p. 375-381.

45. Winter, D.A., A.E. Patla, and J.S. Frank, Assessment of balance control in humans. Medical Progress through Technology, 1990. 16(1-2): p. 31-51.

46. Ring, C., U. Nayak, and B. Isaacs, The effect of visual deprivation and proprioceptive change on postural sway in healthy adults. Journal of the American Geriatrics Society, 1989. 37(8): p. 745-749.

47. Silfies, S.P., J. Cholewicki, and A. Radebold, The effects of visual input on postural control of the lumbar spine in unstable sitting. Human Movement Science, 2003. 22(3): p. 237-252.

48. Riley, M., et al., Common effects of touch and vision on postural parameters. Experimental Brain Research, 1997. 117(1): p. 165-170.

49. Vennila, K. and A.S. Aruin, Postural control in response to a perturbation: role of vision and additional support. Experimental Brain Research, 2011. 212(3): p. 385-397.

50. Woollacott, M.H., A. Shumway-Cook, and L.M. Nashner, Aging and posture control: changes in sensory organization and muscular coordination. The International Journal of Aging and Human Development, 1986. 23(2): p. 97-114.

51. Teasdale, N., G.E. Stelmach, and A. Breunig, Postural sway characteristics of the elderly under normal and altered visual and support surface conditions. Journal of Gerontology, 1991. 46(6): p. B238-B244. 
52. Perry, S.D., L.C. Santos, and A.E. Patla, Contribution of vision and cutaneous sensation to the control of centre of mass (COM) during gait termination. Brain Research, 2001. 913(1): p. 27-34.

53. Hallemans, A., et al., Low vision affects dynamic stability of gait. Gait \& Posture, 2010. 32(4): p. 547-551.

54. Choi, J.-S., et al., Differences in gait pattern between the elderly and the young during level walking under low illumination. Acta of Bioengineering and Biomechanics, 2014. 16(1).

55. Figueiro, M.G., et al., Lighting and perceptual cues: effects on gait measures of older adults at high and low risk for falls. BMC Geriatrics, 2011. 11(1): p. 49.

56. Kesler, A., et al., Shedding light on walking in the dark: the effects of reduced lighting on the gait of older adults with a higher-level gait disorder and controls. Journal of Neuroengineering and Rehabilitation, 2005. 2(1): p. 27.

57. Schmid, M., et al., Equilibrium during static and dynamic tasks in blind subjects: no evidence of cross-modal plasticity. Brain, 2007. 130(8): p. 2097-2107.

58. Nashner, L., Adapting reflexes controlling the human posture. Experimental Brain Research, 1976. 26(1): p. 59-72.

59. Sozzi, S., A. Nardone, and M. Schieppati, Calibration of the leg muscle responses elicited by predictable perturbations of stance and the effect of vision. Frontiers in Human Neuroscience, 2016. 10: p. 419.

60. King, E.C., et al., The use of peripheral vision to guide perturbation-evoked reach-to-grasp balance-recovery reactions. Experimental Brain Research, 2010. 207(1-2): p. 105-118.

61. Ghafouri, M., W.E. McIlroy, and B.E. Maki, Initiation of rapid reach-and-grasp balance reactions: is a pre-formed visuospatial map used in controlling the initial arm trajectory? Experimental Brain Research, 2004. 155(4): p. 532-536.

62. Letts, L., et al., The physical environment as a fall risk factor in older adults: Systematic review and meta-analysis of cross-sectional and cohort studies. Australian Occupational Therapy Journal, 2010. 57(1): p. 51-64.

63. Occupational Safety and Health Administration, Safety and Health Regulations for Construction (1926.56). Retrieved from https://www.osha.gov/lawsregs/regulations/standardnumber/1926/1926.56

64. Occupational Safety and Health Administration, Occupational Safety and Health Standards for Shipyard Employment (1915.82), 2011. Retrieved from https://www.osha.gov/laws-regs/regulations/standardnumber/1915/1915.82

65. International Building Code, Interior Environments: Lighting (1204), 2021. Retrieved from https://codes.iccsafe.org/content/IBC2021P1/chapter-12-interiorenvironment\#IBC2021P1_Ch12_Sec1204

66. Hegde, A.L. and R. Rhodes, Assessment of lighting in independent living facilities and residents' perceptions. Journal of Applied Gerontology, 2010. 29(3): p. 381390.

67. Bakker, R., Y. Iofel, and M.S. Lachs, Lighting levels in the dwellings of homebound older adults. Journal of Housing for the Elderly, 2004. 18(2): p. 1727. 
68. Eilertsen, G., et al., Happy living in darkness! Indoor lighting in relation to activities of daily living, visual and general health in 75-year-olds living at home. Journal of Housing for the Elderly, 2016. 30(2): p. 199-213.

69. Leibrock, C., T. Reuter, and T. Lamb, Molecular basis of dark adaptation in rod photoreceptors. Eye, 1998. 12(3): p. 511-520.

70. Lamb, T. and E.N. Pugh Jr, Dark adaptation and the retinoid cycle of vision. Progress in Retinal and Eye Research, 2004. 23(3): p. 307-380.

71. Van Bommel, W. and G. Van den Beld, Lighting for work: a review of visual and biological effects. Lighting Research \& Technology, 2004. 36(4): p. 255-266.

72. Johnson, E.G. and J.D. Meltzer, Effect of sitting pause times on postural stability after supine-to-standing transfer in dimly lit environments. Journal of Geriatric Physical Therapy, 2012. 35(1): p. 15-19.

73. Burns, E.R., J.A. Stevens, and R. Lee, The direct costs of fatal and non-fatal falls among older adults-United States. Journal of Safety Research, 2016. 58: p. 99103.

74. Owsley, C., Aging and vision. Vision Research, 2011. 51(13): p. 1610-1622.

75. Birren, J.E. and L.M. Fisher. Aging and slowing of behavior: Consequences for cognition and survival. in Psychology and Aging: Nebraska Symposium on Motivation. 1992. University of Nebraska Press, Lincoln, NE.

76. Jackson, G.R., C. Owsley, and G. McGwin Jr, Aging and dark adaptation. Vision Research, 1999. 39(23): p. 3975-3982.

77. Curcio, C., et al., Aging of the human photoreceptor mosaic: evidence for selective vulnerability of rods in central retina. Investigative Ophthalmology \& Visual Science, 1993. 34(12): p. 3278-3296.

78. Hafström, A., et al., Visual influence on postural control, with and without visual motion feedback. Acta Oto-laryngologica, 2002. 122(4): p. 392-397.

79. Rugelj, D., G. Gomišček, and F. Sevšek, The influence of very low illumination on the postural sway of young and elderly adults. PloS One, 2014. 9(8).

80. Masud, T. and R.O. Morris, Epidemiology of falls. Age and Ageing, 2001. 30(suppl 4): p. 3-7.

81. Hignett, $\bar{S}$. and T. Masud, A review of environmental hazards associated with inpatient falls. Ergonomics, 2006. 49(5-6): p. 605-616.

82. Nagano, A., et al., Influence of vision and static stretch of the calf muscles on postural sway during quiet standing. Human Movement Science, 2006. 25(3): p. 422-434.

83. Day, B., et al., Effect of vision and stance width on human body motion when standing: implications for afferent control of lateral sway. The Journal of Physiology, 1993. 469(1): p. 479-499.

84. Maki, B. and G. Ostrovski, Do postural responses to transient and continuous perturbations show similar vision and amplitude dependence? Journal of Biomechanics, 1993. 26(10): p. 1181-1190.

85. McIlroy, W. and B. Maki, Preferred placement of the feet during quiet stance: development of a standardized foot placement for balance testing. Clinical Biomechanics, 1997. 12(1): p. 66-70. 
86. Mcllroy, W. and B. Maki, Adaptive changes to compensatory stepping responses. Gait \& Posture, 1995. 3(1): p. 43-50.

87. Lakhani, B., et al., Characterizing the determinants of limb preference for compensatory stepping in healthy young adults. Gait \& Posture, 2011. 33(2): p. 200-204.

88. Carbonneau, E. and C. Smeesters, Effects of age and lean direction on the threshold of single-step balance recovery in younger, middle-aged and older adults. Gait \& Posture, 2014. 39(1): p. 365-371.

89. Tang, P.-F., M.H. Woollacott, and R.K. Chong, Control of reactive balance adjustments in perturbed human walking: roles of proximal and distal postural muscle activity. Experimental Brain Research, 1998. 119(2): p. 141-152.

90. O'Connor, C.M., et al., Automatic detection of gait events using kinematic data. Gait \& Posture, 2007. 25(3): p. 469-474.

91. Matson, T. and A. Schinkel-Ivy, How does balance during functional tasks change across older adulthood? Gait \& Posture, 2020. 75: p. 34-39.

92. Dempster, W.T., Space requirements of the seated operator, geometrical, kinematic, and mechanical aspects of the body with special reference to the limbs. 1955, Michigan State University.

93. Hof, A.L., The 'extrapolated center of mass' concept suggests a simple control of balance in walking. Human Movement Science, 2008. 27(1): p. 112-125.

94. Salot, P., P. Patel, and T. Bhatt, Reactive balance in individuals with chronic stroke: biomechanical factors related to perturbation-induced backward falling. Physical Therapy, 2016. 96(3): p. 338-347.

95. Patel, P. and T. Bhatt, Adaptation to large-magnitude treadmill-based perturbations: improvements in reactive balance response. Physiological Reports, 2015. 3(2): p. e12247.

96. Patel, P.J. and T. Bhatt, Fall risk during opposing stance perturbations among healthy adults and chronic stroke survivors. Experimental Brain Research, 2018. 236(2): p. 619-628.

97. Nashner, L., The organization of rapid postural adjustments of standing humans: an experimental-conceptual model. Posture and Movement, 1979: p. 243-257.

98. Allum, J., F. Honegger, and H. Schicks, Vestibular and proprioceptive modulation of postural synergies in normal subjects. Journal of Vestibular Research: Equilibrium \& Orientation, 1993. 3(1): p. 59-85.

99. Allum, J. and F. Honegger, Interactions between vestibular and proprioceptive inputs triggering and modulating human balance-correcting responses differ across muscles. Experimental Brain Research, 1998. 121(4): p. 478-494.

100. Madigan, M.L. and E.M. Lloyd, Age and stepping limb performance differences during a single-step recovery from a forward fall. The Journals of Gerontology Series A: Biological Sciences and Medical Sciences, 2005. 60(4): p. 481-485.

101. Patla, A.E. and M. Greig, Any way you look at it, successful obstacle negotiation needs visually guided on-line foot placement regulation during the approach phase. Neuroscience Letters, 2006. 397(1-2): p. 110-114. 
102. Mohagheghi, A.A., R. Moraes, and A.E. Patla, The effects of distant and on-line visual information on the control of approach phase and step over an obstacle during locomotion. Experimental Brain Research, 2004. 155(4): p. 459-468.

103. Forghani, A., R. Preuss, and T. Milner, Postural response characterization of standing humans to multi-directional, predictable and unpredictable perturbations to the arm. Journal of Electromyography and Kinesiology, 2017. 32: p. 83-92.

104. Lin, S.-I. and M.H. Woollacott, Postural muscle responses following changing balance threats in young, stable older, and unstable older adults. Journal of Motor Behavior, 2002. 34(1): p. 37-44.

105. McIlroy, W. and B. Maki, Do anticipatory postural adjustments precede compensatory stepping reactions evoked by perturbation? Neuroscience Letters, 1993. 164(1-2): p. 199-202.

106. Dietz, V., G. Horstmann, and W. Berger, Interlimb coordination of leg-muscle activation during perturbation of stance in humans. Journal of Neurophysiology, 1989. 62(3): p. 680-693.

107. Allum, J. and C. Pfaltz, Visual and vestibular contributions to pitch sway stabilization in the ankle muscles of normals and patients with bilateral peripheral vestibular deficits. Experimental Brain Research, 1985. 58(1): p. 8294.

108. Criswell, E., Cram's introduction to surface electromyography. 2010: Jones \& Bartlett Publishers.

109. Schinkel-Ivy, A. and C.A. Duncan, The effects of short-term and long-term experiences on co-contraction of lower extremity postural control muscles during continuous, multi-directional support-surface perturbations. Journal of Electromyography and Kinesiology, 2018. 39: p. 42-48.

110. Di Fabio, R.P., Reliability of computerized surface electromyography for determining the onset of muscle activity. Physical Therapy, 1987. 67(1): p. 43-48.

111. Nelson-Wong, E. and J.P. Callaghan, Is muscle co-activation a predisposing factor for low back pain development during standing? A multifactorial approach for early identification of at-risk individuals. Journal of Electromyography and Kinesiology, 2010. 20(2): p. 256-263.

112. Schinkel-Ivy, A., B.C. Nairn, and J.D. Drake, Investigation of trunk muscle cocontraction and its association with low back pain development during prolonged sitting. Journal of Electromyography and Kinesiology, 2013. 23(4): p. 778-786.

113. Lewek, M.D., K.S. Rudolph, and L. Snyder-Mackler, Control of frontal plane knee laxity during gait in patients with medial compartment knee osteoarthritis. Osteoarthritis and Cartilage, 2004. 12(9): p. 745-751.

114. Granata, K., et al., Active stiffness of the ankle in response to inertial and elastic loads. Journal of Electromyography and Kinesiology, 2004. 14(5): p. 599-609.

115. Lee, P.J., E.L. Rogers, and K.P. Granata, Active trunk stiffness increases with cocontraction. Journal of Electromyography and Kinesiology, 2006. 16(1): p. 51-57.

116. Nelson-Wong, E., et al., Application of autocorrelation and cross-correlation analyses in human movement and rehabilitation research. Journal of Orthopaedic \& Sports Physical Therapy, 2009. 39(4): p. 287-295. 
117. Sozzi, S., et al., Leg muscle activity during tandem stance and the control of body balance in the frontal plane. Clinical Neurophysiology, 2013. 124(6): p. 11751186.

118. Horak, F.B., L.M. Nashner, and H. Diener, Postural strategies associated with somatosensory and vestibular loss. Experimental Brain Research, 1990. 82(1): p. 167-177.

119. Allum, J. and K. Mauritz, Compensation for intrinsic muscle stiffness by shortlatency reflexes in human triceps surae muscles. Journal of Neurophysiology, 1984. 52(5): p. 797-818.

120. Inglis, J.T., et al., The importance of somatosensory information in triggering and scaling automatic postural responses in humans. Experimental Brain Research, 1994. 101(1): p. 159-164.

121. Horak, F.B. and L.M. Nashner, Central programming of postural movements: adaptation to altered support-surface configurations. Journal of Neurophysiology, 1986. 55(6): p. 1369-1381.

122. Lee, P.-Y., et al., Reactive balance control in older adults with diabetes. Gait \& Posture, 2018. 61: p. 67-72.

123. Pavol, M.J., et al., Age influences the outcome of a slipping perturbation during initial but not repeated exposures. The Journals of Gerontology Series A: Biological Sciences and Medical Sciences, 2002. 57(8): p. M496-M503.

124. Maki, B.E. and W.E. McIlroy, Control of rapid limb movements for balance recovery: age-related changes and implications for fall prevention. Age and Ageing, 2006. 35(suppl_2): p. ii12-ii18.

125. Wolfson, L., et al., Stressing the postural response: a quantitative method for testing balance. Journal of the American Geriatrics Society, 1986. 34(12): p. 845850.

126. Luchies, C.W., et al., Stepping responses of young and old adults to postural disturbances: kinematics. Journal of the American Geriatrics Society, 1994. 42(5): p. 506-512.

127. Mansfield, A., et al., Is impaired control of reactive stepping related to falls during inpatient stroke rehabilitation? Neurorehabilitation and neural repair, 2013. 27(6): p. 526-533.

128. Inness, E.L., et al., Impaired reactive stepping among patients ready for discharge from inpatient stroke rehabilitation. Physical therapy, 2014. 94(12): p. 1755-1764.

129. Thelen, D.G., et al., Age differences in using a rapid step to regain balance during a forward fall. The Journals of Gerontology Series A: Biological Sciences and Medical Sciences, 1997. 52(1): p. M8-M13.

130. International Building Code, Interior Environments: Lighting (1204), 2021. Retrieved from https://codes.iccsafe.org/content/IBC2021P1/chapter-12-interiorenvironment\#IBC2021P1_Ch12_Sec1204

131. Gill, T.M., et al., A population-based study of environmental hazards in the homes of older persons. American Journal of Public Health, 1999. 89(4): p. 553-556. 


\section{A Participant Eligibility Checklist}

\begin{tabular}{|l|l|l|}
\hline $\begin{array}{l}\text { Site: Neuromechanics } \\
\text { Lab, SDC 121 }\end{array}$ & IRB ID:1433509-2 & $\begin{array}{l}\text { Study Title: Evaluating the } \\
\text { Influence of Lighting Conditions on } \\
\text { Balance Recovery }\end{array}$ \\
\hline $\begin{array}{l}\text { Principal } \\
\text { Investigator: Dr. }\end{array}$ & $\begin{array}{l}\text { Protocol } \\
\text { Carolyn Duncan }\end{array}$ & \\
\hline $\begin{array}{l}\text { Participant } \\
\text { Identification } \\
\text { Number: }\end{array}$ & Version: & \\
\hline
\end{tabular}

For each inclusion and exclusion criterion listed below from the study protocol, indicate whether the participant has met the criterion and document their overall participant eligibility to participate in the clinical trial.

\begin{tabular}{|c|c|c|}
\hline \multicolumn{3}{|c|}{ INCLUSION CRITERIA } \\
\hline $\begin{array}{l}\text { Criterion } \\
\text { number }\end{array}$ & Description & $\begin{array}{l}\mathrm{Yes} / \mathrm{No} / \text { or } \\
\quad \mathrm{N} / \mathrm{A} ?\end{array}$ \\
\hline 1 & Are between $18-40$ years of age & Yes No \\
\hline \multicolumn{3}{|c|}{ EXCLUSION CRITERIA } \\
\hline $\begin{array}{l}\text { Criterion } \\
\text { number }\end{array}$ & Description & $\begin{array}{l}\mathrm{Yes} / \mathrm{No} / \text { or } \\
\quad \mathrm{N} / \mathrm{A} ?\end{array}$ \\
\hline 1 & Weight $>300 \mathrm{lbs}$ & Yes No \\
\hline 2 & $\begin{array}{c}\text { Has been diagnosed or is diagnosed with osteoporosis, } \\
\text { neck injuries, head injuries, any injuries to the lower } \\
\text { body, sacroiliac injuries, or any history of compression } \\
\text { fracture of the vertebral body }\end{array}$ & Yes No \\
\hline 3 & $\begin{array}{c}\text { Has been diagnosed or is diagnosed with a traumatic } \\
\text { brain injury }\end{array}$ & Yes $\quad$ No \\
\hline 4 & Has been in a previous lean-and-release study & Yes No \\
\hline
\end{tabular}

Inclusion/Exclusion Criteria Documented by: Date:

Printed Name

yyyy $/ \mathrm{mm} / \mathrm{dd}$

Investigator Assessment:

All required tests and procedures have been completed. Based on my review, this participant is:

○ Eligible to participate in the study

- Not eligible to participate in the study

Completed by: Date: 


\section{B Medical History Questionnaire}

\begin{tabular}{|l|c|c|}
\hline Subject Information & Circle & Exclude? \\
\hline Are you right or left handed? & R L both & \\
\hline Which hand do you write with? & R L both & \\
\hline With which foot would you kick a ball? & R L both & \\
\hline Have you ever participated in any postural balance study before? & Y N & \\
\hline Have you ever participated in a moving floor study? & Y N & \\
\hline
\end{tabular}

\begin{tabular}{|l|l|l|l|l|}
\hline General Questions & Circle & Describe & Exclude? \\
\hline $\begin{array}{l}\text { In the past year have you had any } \\
\text { episodes where you felt dizzy, unsteady, or } \\
\text { weak? }\end{array}$ & Y N & & \\
\hline Are you susceptible to motion sickness? & Y N N & & \\
\hline $\begin{array}{l}\text { Do you have any conditions that limit the } \\
\text { use of your arms and legs? }\end{array}$ & Y N & & \\
\hline $\begin{array}{l}\text { Do you have any vision problems that limit } \\
\text { your ability to read, watch TV, drive a car, } \\
\text { or do any other activities }\end{array}$ & Y N & & \\
\hline
\end{tabular}

I am going to list a number of medical conditions, and I would like you to tell me if you have ever been diagnosed with any of them. Please remember that you are free to choose not to respond to any of these questions. If you do not wish to respond to a question simply tell me that you pass on the question.

\begin{tabular}{|l|l|l|l|}
\hline $\begin{array}{l}\text { Do you have or have } \\
\text { you ever had: }\end{array}$ & Circle & \\
\hline Paralysis & Y & N & \\
\hline Epilepsy & Y & N & \\
\hline Cerebral palsy & Y & N & \\
\hline Multiple Sclerosis & Y & N & \\
\hline Parkinson's disease & Y & N & \\
\hline A Stroke & Y & N & \\
\hline Diabetes & Y & N & \\
\hline Peripheral Neuropathy & Y & N & \\
\hline Depression & Y & N & \\
\hline $\begin{array}{l}\text { Any other neurological } \\
\text { disorder }\end{array}$ & Y & N & \\
\hline $\begin{array}{l}\text { A vision problem that is } \\
\text { not corrected by glasses } \\
\text { e.g glaucoma }\end{array}$ & Y & N & \\
\hline $\begin{array}{l}\text { Cataracts or cataract } \\
\text { surgery }\end{array}$ & Y & N & \\
\hline $\begin{array}{l}\text { A balance or coordination } \\
\text { problem }\end{array}$ & Y & N & \\
\hline $\begin{array}{l}\text { An inner ear (vestibular) } \\
\text { problem }\end{array}$ & Y & N & \\
\hline
\end{tabular}




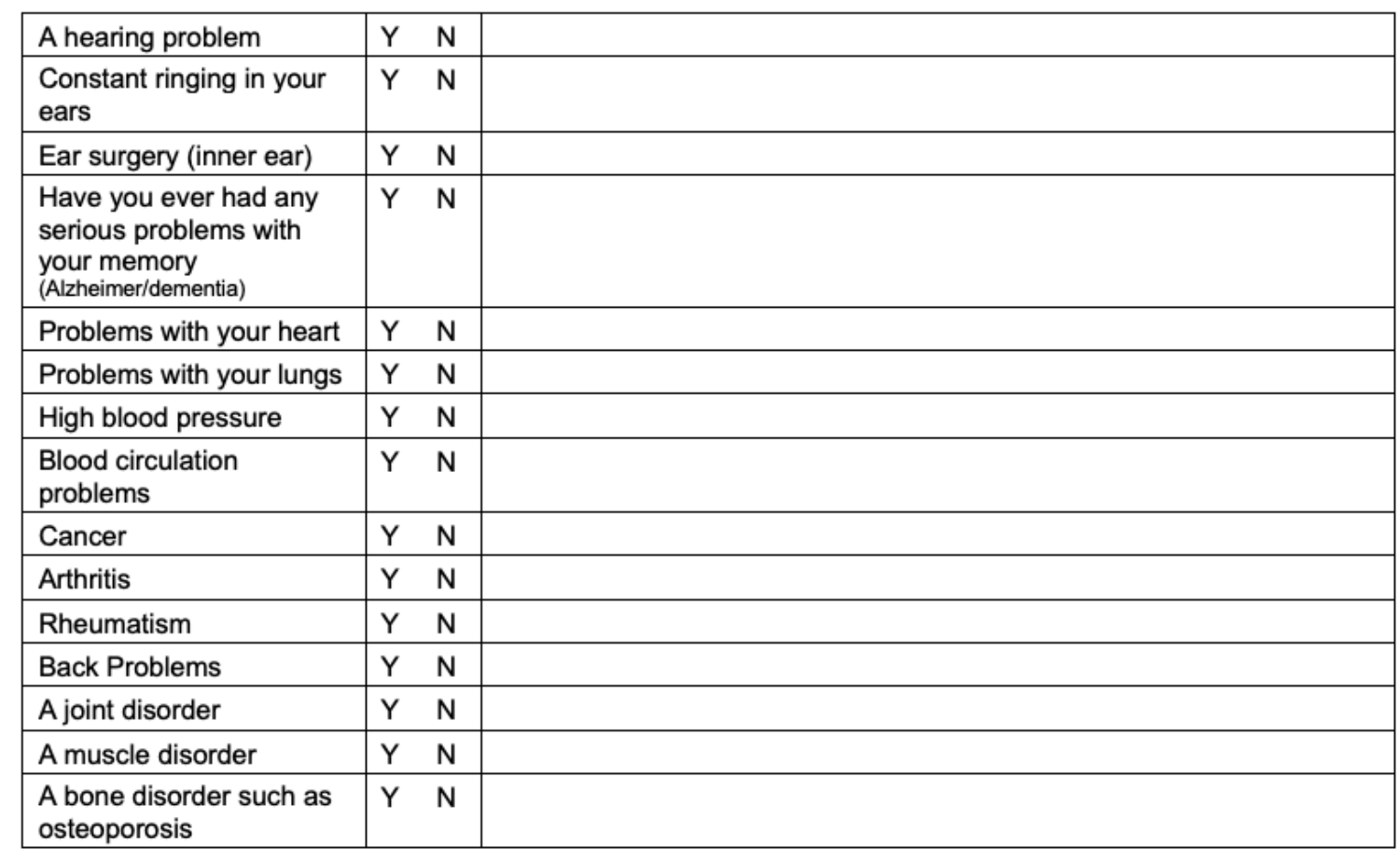

\begin{tabular}{|l|l|l|l|}
\hline $\begin{array}{l}\text { Have you ever severely } \\
\text { injured or had surgery on } \\
\text { your: }\end{array}$ & Circle & \\
\hline Head & Y & N & \\
\hline Neck & Y & N & \\
\hline Back & Y & N & \\
\hline Pelvis & Y & N & \\
\hline Ankle & Y & N & \\
\hline Knee & Y & N & \\
\hline Hip & Y & N & \\
\hline $\begin{array}{l}\text { Do you have any prosthetic } \\
\text { limbs? }\end{array}$ & Y & N & \\
\hline $\begin{array}{l}\text { Have you had a joint } \\
\text { replacement or a joint } \\
\text { fusion? }\end{array}$ & Y & N & \\
\hline $\begin{array}{l}\text { Have you ever broken any } \\
\text { bones? }\end{array}$ & Y & N & \\
\hline
\end{tabular}

\begin{tabular}{|l|c|l|l|}
\hline $\begin{array}{l}\text { In the PAST MONTH have } \\
\text { you had any: }\end{array}$ & Circle & Describe \\
\hline IIInesses & Y & N & \\
\hline Injuries & Y & N & \\
\hline Operations & Y & N & \\
\hline
\end{tabular}


List all medications (prescription or over-the-counter) that you are currently taking?

\begin{tabular}{|l|l|l|}
\hline Medication/dosage & Ailment (disease) & Frequency of use \\
\hline & & \\
\hline & & \\
\hline & & \\
\hline & & \\
\hline & & \\
\hline \multicolumn{2}{|l|}{ Have any of these drugs made you feel dizzy, unsteady, or weak? } \\
\hline
\end{tabular}

\begin{tabular}{|l|l|}
\hline PAR-Q & \\
\hline $\begin{array}{l}\text { 1. Has your doctor ever said that you have a heart condition AND that you should only do } \\
\text { physical activity recommended by a primary care physician? }\end{array}$ & Yes \\
\hline 2. Do you feel pain in your chest when you do physical activity? & Yes \\
\hline 3. In the past month, have you had chest pain when you were not doing physical activity? & Yes No \\
\hline 4. Do you lose your balance because of dizziness or do you ever lose consciousness? & Yes No \\
\hline $\begin{array}{l}\text { 5. Do you have a bone or joint problem (for example, back, knee or hip) that could be made } \\
\text { worse by a change in your physical activity? }\end{array}$ & Yes No \\
\hline $\begin{array}{l}\text { 6. Is your doctor currently prescribing drugs (for example, water pills) for your blood } \\
\text { pressure or heart condition? }\end{array}$ & Yes No \\
\hline 7. Do you know of ANY OTHER REASON why you should not do physical activity? & Yes \\
\hline
\end{tabular}

***Yes answer on any PAR-Q question - will be excluded from the study *** 


\section{Lean Angle Data}

\begin{tabular}{cccc}
\hline Participant & \multicolumn{2}{c}{ Max Lean Angle $\mathbf{(}^{\mathbf{1}} \mathbf{1}^{\mathbf{2}}$} & Max Successful Lean Angle $\mathbf{(}^{\mathbf{2}} \mathbf{~}^{\mathbf{2}}$ \\
\cline { 2 - 4 } & Light & Dark & 20 \\
\hline 1 & 30 & 22 & 40 \\
2 & 44 & 42 & 28 \\
3 & 30 & 30 & 36 \\
4 & 38 & 38 & 24 \\
5 & 26 & 34 & 30 \\
6 & 44 & 32 & 30 \\
7 & 34 & 32 & 28 \\
8 & 34 & 30 & 18 \\
9 & 24 & 20 & 28 \\
10 & 30 & 32 & 28 \\
11 & 30 & 32 & 22 \\
12 & 24 & 30 & 30 \\
13 & 34 & 32 & 28 \\
14 & 30 & 32 & 18 \\
15 & 26 & 20 & 14 \\
16 & 16 & 20 & 20 \\
17 & 24 & 22 & 18 \\
18 & 24 & 20 & 18 \\
19 & 20 & 24 & 22 \\
20 & 28 & 24 & $25.0(6.7)$ \\
\hline Mean (SD) & $29.5(7.1)$ & $28.4(6.5)$ & \\
\hline
\end{tabular}

${ }^{1}$ Lean angle where balance could not be recovered with 1 step in 3 out of 5 attempts

${ }^{2}$ Highest lean angle where balance could be recovered with 1 step in both lighting conditions 
D Paper 1: Paired T-Test Results

\begin{tabular}{|c|c|c|}
\hline Outcome & Paired t-test & $\begin{array}{l}\text { Effect Size } \\
\text { (Cohen's d) }\end{array}$ \\
\hline Maximum Lean Angle & $t(19)=1.02, p=0.32$ & 0.23 \\
\hline \multicolumn{3}{|l|}{ Stepping Parameters } \\
\hline Step Length & $t(19)=-2.26, p=0.04$ & -0.50 \\
\hline Step Width & $t(19)=-0.62, p=0.54$ & -0.14 \\
\hline Step Height & $t(19)=-0.97, p=0.34$ & -0.22 \\
\hline Step Velocity & $t(19)=-1.39, p=0.18$ & -0.31 \\
\hline Step Time & $t(19)=-1.36, p=0.19$ & -0.30 \\
\hline Step Onset Time & $t(19)=0.96, p=0.35$ & 0.21 \\
\hline \multicolumn{3}{|l|}{ COM Kinematics } \\
\hline COM Displacement: A-P & $t(19)=-1.30, p=0.21$ & -0.29 \\
\hline COM Displacement: M-L & $t(19)=-0.56, p=0.58$ & -0.12 \\
\hline Peak COM Velocity: A-P & $t(19)=-0.13, p=0.90$ & -0.03 \\
\hline Peak COM Velocity: M-L & $t(19)=-1.33, p=0.20$ & -0.30 \\
\hline Peak COM Acceleration: A-P & $t(19)=-1.42, p=0.17$ & -0.32 \\
\hline Peak COM Acceleration: M-L & $t(19)=0.39, p=0.70$ & 0.09 \\
\hline \multicolumn{3}{|l|}{ Postural Stability } \\
\hline$A-P$ & $t(19)=2.19, p=0.04$ & 0.49 \\
\hline$M-L$ & $t(19)=-0.99, p=0.34$ & -0.22 \\
\hline \multicolumn{3}{|l|}{ Angular Kinematics } \\
\hline Ankle Dorsiflexion & $t(19)=-0.81, p=0.43$ & -0.18 \\
\hline Ankle Plantarflexion & $t(19)=0.93, p=0.37$ & 0.21 \\
\hline Knee Flexion & $t(18)=-0.26, p=0.02$ & -0.60 \\
\hline Knee Extension & $t(19)=2.47, p=0.02$ & 0.55 \\
\hline Hip Flexion & $\mathrm{t}(19)=-2.09, p=0.05$ & -0.47 \\
\hline Hip Extension & $t(16)=1.15, p=0.27$ & 0.28 \\
\hline Trunk Flexion & $t(18)=-1.89, p=0.08$ & -0.43 \\
\hline Trunk Extension & $t(18)=3.31, p<0.01$ & 0.81 \\
\hline
\end{tabular}




\section{E Paper 2: Paired T-Test Results}

\begin{tabular}{|c|c|c|}
\hline Outcome & Paired t-test & $\begin{array}{l}\text { Effect Size } \\
\text { (Cohen's d) }\end{array}$ \\
\hline \multicolumn{3}{|l|}{ Muscle Onset Latencies } \\
\hline TA: Stepping Leg & $t(17)=-0.69, p=0.50$ & -0.16 \\
\hline TA: Standing Leg & $t(15)=1.18, p=0.26$ & 0.29 \\
\hline MG: Stepping Leg & $t(11)=0.89, p=0.40$ & 0.26 \\
\hline MG: Standing Leg & $t(10)=-0.04, p=0.97$ & -0.01 \\
\hline RF: Stepping Leg & $t(18)=0.10, p=0.92$ & 0.02 \\
\hline RF: Standing Leg & $t(17)=0.21, p=0.84$ & 0.05 \\
\hline BF: Stepping Leg & $t(15)=0.51, p=0.62$ & 0.13 \\
\hline BF: Standing Leg & $t(16)=0.15, p=0.89$ & 0.04 \\
\hline \multicolumn{3}{|c|}{ Mean Muscle Activations: Pre-Perturbation } \\
\hline TA: Stepping Leg & $t(18)=1.76, p=0.10$ & 0.40 \\
\hline TA: Standing Leg & $t(18)=0.81, p=0.43$ & 0.18 \\
\hline MG: Stepping Leg & $t(18)=0.17, p=0.87$ & 0.04 \\
\hline MG: Standing Leg & $t(18)=0.35, p=0.73$ & 0.08 \\
\hline RF: Stepping Leg & $t(18)=0.43, p=0.67$ & 0.10 \\
\hline RF: Standing Leg & $t(18)=1.64, p=0.12$ & 0.38 \\
\hline BF: Stepping Leg & $t(18)=0.05, p=0.96$ & 0.01 \\
\hline BF: Standing Leg & $t(17)=-1.35, p=0.19$ & 0.32 \\
\hline \multicolumn{3}{|c|}{ Mean Muscle Activations: Post-Perturbation } \\
\hline TA: Stepping Leg & $t(18)=-1.17, p=0.26$ & -0.27 \\
\hline TA: Standing Leg & $t(18)=-2.26, p=0.04$ & -0.52 \\
\hline MG: Stepping Leg & $t(18)=-1.12, p=0.28$ & -0.26 \\
\hline MG: Standing Leg & $t(18)=0.84, p=0.41$ & 0.19 \\
\hline RF: Stepping Leg & $t(18)=-0.99, p=0.33$ & -0.23 \\
\hline RF: Standing Leg & $t(18)=-1.43, p=0.17$ & -0.33 \\
\hline BF: Stepping Leg & $t(18)=-0.99, p=0.34$ & -0.22 \\
\hline BF: Standing Leg & $t(17)=-0.69, p=0.50$ & -0.16 \\
\hline
\end{tabular}




\begin{tabular}{ccc}
\hline Outcome & Paired t-test & $\begin{array}{c}\text { Effect Size } \\
\text { (Cohen's d) }\end{array}$ \\
\hline Maximum Muscle Activations: Pre-Perturbation & \\
TA: Stepping Leg & $\mathrm{t}(18)=-0.31, \mathrm{p}=0.76$ & -0.07 \\
TA: Standing Leg & $\mathrm{t}(18)=1.00, \mathrm{p}=0.33$ & 0.23 \\
MG: Stepping Leg & $\mathrm{t}(18)=0.10, \mathrm{p}=0.92$ & 0.02 \\
MG: Standing Leg & $\mathrm{t}(18)=1.09, \mathrm{p}=0.29$ & 0.25 \\
RF: Stepping Leg & $\mathrm{t}(18)=-1.18, \mathrm{p}=0.25$ & -0.27 \\
RF: Standing Leg & $\mathrm{t}(18)=1.40, \mathrm{p}=0.18$ & 0.32 \\
BF: Stepping Leg & $\mathrm{t}(18)=0.05, \mathrm{p}=0.96$ & 0.01 \\
BF: Standing Leg & $\mathrm{t}(17)=-1.28, \mathrm{p}=0.22$ & -0.30 \\
\hline MA: Stepping Leg & $\mathrm{t}(18)=-1.14, \mathrm{p}=0.27$ & -0.26 \\
TA: Standing Leg & $\mathrm{t}(18)=-1.42, \mathrm{p}=0.17$ & -0.34 \\
MG: Stepping Leg & $\mathrm{t}(18)=-0.45, \mathrm{p}=0.66$ & -0.10 \\
MG: Standing Leg & $\mathrm{t}(18)=0.94, \mathrm{p}=0.36$ & 0.22 \\
RF: Stepping Leg & $\mathrm{t}(18)=-0.38, \mathrm{p}=0.71$ & -0.09 \\
RF: Standing Leg & $\mathrm{t}(18)=-1.97, \mathrm{p}=0.06$ & -0.45 \\
BF: Stepping Leg & $\mathrm{t}(18)=-0.41, \mathrm{p}=0.69$ & -0.09 \\
BF: Standing Leg & $\mathrm{t}(18)=-1.24, \mathrm{p}=0.23$ & -0.29 \\
\hline
\end{tabular}




\begin{tabular}{ccc}
\hline Outcome & Paired t-test & $\begin{array}{c}\text { Effect Size } \\
\text { (Cohen's d) }\end{array}$ \\
\hline Co-Contraction Index (CCI) & & \\
TA-MG: Stepping Leg & $\mathrm{t}(18)=-0.61, \mathrm{p}=0.55$ & -0.14 \\
TA-MG: Standing Leg & $\mathrm{t}(18)=-0.22, \mathrm{p}=0.83$ & -0.05 \\
RF-BF: Stepping Leg & $\mathrm{t}(18)=-1.24, \mathrm{p}=0.23$ & -0.28 \\
RF-BF: Standing Leg & $\mathrm{t}(17)=1.02, \mathrm{p}=0.32$ & 0.24 \\
TA-RF: Stepping Leg & $\mathrm{t}(18)=1.09, \mathrm{p}=0.29$ & 0.25 \\
TA-RF: Standing Leg & $\mathrm{t}(18)=1.80, \mathrm{p}=0.09$ & 0.41 \\
MG-BF: Stepping Leg & $\mathrm{t}(17)=-1.50, \mathrm{p}=0.15$ & -0.35 \\
MG-BF: Standing Leg & $\mathrm{t}(16)=0.08, \mathrm{p}=0.94$ & 0.02 \\
\hline TA-MG: Stepping Leg & $\mathrm{t}(18)=1.32, \mathrm{p}=0.20$ & 0.30 \\
TA-MG: Standing Leg & $\mathrm{t}(18)=1.44, \mathrm{p}=0.17$ & 0.33 \\
RF-BF: Stepping Leg & $\mathrm{t}(18)=-0.38, \mathrm{p}=0.71$ & -0.09 \\
RF-BF: Standing Leg & $\mathrm{t}(17)=2.59, \mathrm{p}=0.02$ & 0.61 \\
TA-RF: Stepping Leg & $\mathrm{t}(18)=0.54, \mathrm{p}=0.60$ & 0.12 \\
TA-RF: Standing Leg & $\mathrm{t}(18)=0.60, \mathrm{p}=0.56$ & 0.14 \\
MG-BF: Stepping Leg & $\mathrm{t}(18)=-0.95, \mathrm{p}=0.35$ & -0.22 \\
MG-BF: Standing Leg & $\mathrm{t}(17)=1.01, \mathrm{p}=0.33$ & 0.24 \\
\hline
\end{tabular}

